TRANSACTIONS OF THE

AMERICAN MATHEMATICAL SOCIETY

Volume 356, Number 6, Pages 2515-2543

S 0002-9947(04)03447-6

Article electronically published on January 21, 2004

\title{
AUTOMORPHISMS OF SUBFACTORS FROM COMMUTING SQUARES
}

\author{
ANNE LOUISE SVENDSEN
}

\begin{abstract}
We study an infinite series of irreducible, hyperfinite subfactors, which are obtained from an initial commuting square by iterating Jones' basic construction. They were constructed by Haagerup and Schou and have $A_{\infty}$ as principal graphs, which means that their standard invariant is "trivial". We use certain symmetries of the initial commuting squares to construct explicitly non-trivial outer automorphisms of these subfactors. These automorphisms capture information about the subfactors which is not contained in the standard invariant.
\end{abstract}

\section{INTRODUCTION}

The theory of subfactors was founded by Jones in [8]. He introduced the first invariants for inclusions of $\mathrm{II}_{1}$ factors $N \subset M$, namely the Jones index and the principal graphs, and showed that if the index is less than 4 , then it has to be of the form $4 \cos ^{2} \frac{\pi}{n}$ for some $n \geq 3$. In this case the principal graphs are Coxeter-Dynkin graphs of type A, D, or E. It turns out that in general the principal graphs do not determine the subfactor. A finer invariant, the so-called standard invariant, of a subfactor $N \subset M$ of finite Jones index is obtained as follows ([8], see also [5]): By iterating the basic construction for $N \subset M$ we obtain a tower of $\mathrm{II}_{1}$ factors $N \subset M \subset M_{1} \subset M_{2} \subset \cdots$ canonically associated to $N \subset M$. The trace-preserving isomorphism class of the following sequence of commuting squares of higher relative commutants

$$
\begin{aligned}
& \mathbb{C}=M^{\prime} \cap M=M^{\prime} \cap M_{1} \subset M^{\prime} \cap M_{2} \subset M^{\prime} \cap M_{3} \subset \ldots \\
& \cup \cup \cup \\
& \mathbb{C}=M_{1}^{\prime} \cap M_{1} \subset M_{1}^{\prime} \cap M_{2} \quad \subset \quad M_{1}^{\prime} \cap M_{3} \quad \subset \quad \cdots
\end{aligned}
$$

is then the standard invariant for $N \subset M$.

One of the main problems in the theory of subfactors is the classification of subfactors of the hyperfinite $\mathrm{II}_{1}$ factor $R$. To this end Popa introduced a concept of amenability for subfactors and proved that the standard invariant is a complete invariant for amenable subfactors of $R$ [16. If the (hyperfinite) subfactor is not amenable, then invariants other than the standard invariant are likely to become important. In particular, if the Jones index $[M: N]$ is larger than 4 and the princi-

Received by the editors December 9, 2002 and, in revised form, June 2, 2003.

2000 Mathematics Subject Classification. Primary 46L37, 46L40.

(C)2004 American Mathematical Society 
pal graphs are $A_{\infty}$ (this means that the standard invariant is "trivial", i.e. consists of just the Temperley-Lieb algebras), then it is not clear how to capture properties of such a subfactor. One attempt to try to do this is to study the group of subfactor automorphisms, i.e. outer automorphisms of $M$, which leave the subfactor $N$ globally invariant.

In this paper we study an infinite series of irreducible, hyperfinite subfactors of this type. Their Jones indices lie between 4 and 4.5 and they have principal graphs $A_{\infty}$. These subfactors were previously obtained by Haagerup and Schou by constructing a non-degenerate commuting square and iterating Jones' basic construction for the multi-matrix algebras in the commuting square [18. The initial commuting squares have some very nice symmetries, which are however no longer present in the standard invariant, since it is trivial (in the above sense). It turns out that these symmetries can be used to construct explicitly outer automorphisms of order two for this infinite series of Haagerup-Schou subfactors. Note that work on automorphisms of subfactors has been done by several authors; see for instance [6], 11], [12, to mention just a few articles relevant for the work below.

A more detailed description of the paper follows. Section 2 is a review of a general method for constructing an automorphism of a subfactor by using the symmetries of the inclusion graphs of the initial commuting square to construct a symmetric connection. We work in the string algebra representation and use Ocneanu's biunitary condition to describe the initial non-degenerate commuting square (13, [14]). We use the notation from Haagerup and Schou [18. Using the string algebra representation we consider graph automorphisms of the four individual inclusion graphs, which preserve Perron-Frobenius weights and match with the structure of the commuting square, i.e. respects the identification of the vertices in the commuting square. For such a collection of graph automorphisms to give rise to an automorphism of the commuting square, the unitary matrix which gives the connection and has entries labeled by the four graphs, has to be invariant under the map arising from the graph automorphisms, i.e. the connection has to be symmetric. When this is the case the graph automorphism gives an automorphism of the commuting square, which extends to an automorphisms of the subfactor. Under a mild condition this subfactor automorphism is outer. The results in this section are well known; see for instance [6].

In section 3 we investigate the series of Haagerup-Schou subfactors. They are constructed from initial non-degenerate commuting squares, which have the graph shown in Figure 1 as one of the inclusion graphs.

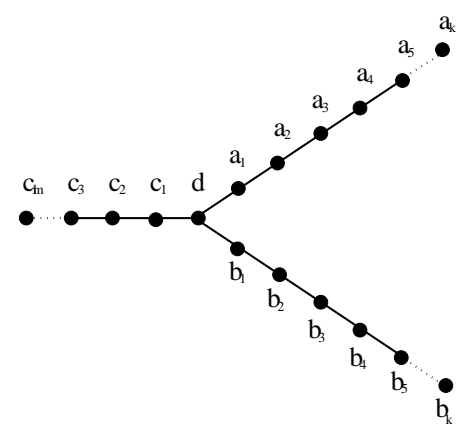

FIGURE 1. 
We consider the series of examples where $m=k-1$ for $k \geq 3$. The symmetry we use is the graph automorphism which switches the $a$ - and $b$-rays. We compute explicitly a symmetric connection for the infinite series of examples, in the course of which we have to show several polynomial identities. Our computations are similar to commuting square computations performed by Haagerup and Schou, but they are more special since we want to construct a symmetric connection. This means that the entries of the connection have to satisfy many more identities. (Observe that it is in general not true that these identities have solutions). It then follows that we have constructed an outer automorphism of order two of each of the subfactors in the infinite series of Haagerup-Schou subfactors. The construction of an outer automorphism of order two can also be carried out using the same method for each of the subfactors in the infinite series of examples of Haagerup-Schou subfactors with $m=k-2$ for $k \geq 4$ [19].

\section{Construction of automorphisms of Subfactors VIA SYMMETRIC CONNECTIONS}

In this section we review a method that leads to the construction of an automorphism of a subfactor from symmetries of the inclusion graphs by construting a symmetric connection.

We consider the following situation. Let $A_{00} \subset A_{01} \subset A_{11}$, and $A_{00} \subset A_{10} \subset$ $A_{11}$ be inclusions of four finite-dimensional $C^{*}$-algebras with inclusion matrices $G, K, L$, and $H$ as follows:

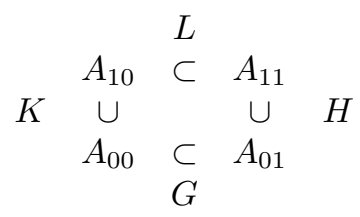

The algebras are finite-dimensional $\mathrm{C}^{*}$-algebras, hence they are direct sums of matrix algebras. Let $M_{n m}(\mathbb{Z})$ denote the $n \times m$ matrices over $\mathbb{Z}$. Since $G, H, K$, and $L$ are inclusion matrices we have that $G \in M_{n m}(\mathbb{Z}), L \in M_{p q}(\mathbb{Z}), K \in M_{n p}(\mathbb{Z})$, and $H \in M_{m q}(\mathbb{Z})$ are matrices with non-negative entries, where $n$ denotes the number of simple summands of $A_{00}, m$ denotes the number of simple summands of $A_{01}, p$ denotes the number of simple summands of $A_{10}$, and $q$ denotes the number of simple summands of $A_{11}$. Let $\Gamma_{X}$ be the bipartite graph corresponding to the matrix $X$, i.e. the graph with adjacency matrix

$$
\Delta_{\Gamma_{X}}=\left(\begin{array}{rr}
0 & X \\
X^{t} & 0
\end{array}\right) .
$$

For an inclusion matrix $X$ we let $\left(\Gamma_{X}\right)_{\text {even }}$ be the vertices labeling the rows of $X$ and $\left(\Gamma_{X}\right)_{\text {odd }}$ be the vertices labeling the columns of $X$. Since both $\left(\Gamma_{K}\right)_{\text {even }}$ and $\left(\Gamma_{G}\right)_{\text {even }}$ label the simple summands of $A_{00}$, we have an identification between the even vertices of the bipartite graphs $\Gamma_{K}$ and $\Gamma_{G}$. Similarly the odd vertices of $\Gamma_{G}$ are identified with the even vertices of $\Gamma_{H}$, the odd vertices of $\Gamma_{K}$ are identified with the even vertices of $\Gamma_{L}$, and the odd vertices of $\Gamma_{L}$ are identified with the odd vertices of $\Gamma_{H}$. We will suppose that all the graphs $\Gamma_{G}, \Gamma_{H}, \Gamma_{K}$, and $\Gamma_{L}$ are connected. Recall that the graph $\Gamma_{X}$ is connected if and only if the matrix $X X^{t}$ is irreducible, if and only if the adjacency matrix $\Delta_{\Gamma_{X}}$ is irreducible [5]. 
Let $\tau$ be a fixed normalized trace on $A_{11}$. Then (2.1) is a commuting square with respect to $\tau$ if $E_{A_{01}} E_{A_{10}}=E_{A_{00}}$, where $E_{X}$ denotes the unique trace-preserving conditional expectation onto the algebra $X$ [5]. It is clearly necessary that $G H=$ $K L$, but we will furthermore require that $G^{t} K=H L^{t}$, which is equivalent to requiring that $A_{01} \cdot A_{10}$ linearly spans $A_{11}$. A commuting square satisfying this condition is called non-degenerate [16], [18]. Recall that the concept of a commuting square was introduced by Popa in [15. Throughout this section we work with a fixed normalized trace $\tau$ on $A_{11}$ and assume that the inclusion of the four finitedimensional algebras in (2.1) is a non-degenerate commuting square.

2.1. Commuting squares, the bi-unitary condition. From now on let us assume that $A_{00}$ is abelian, i.e. $A_{00} \cong \mathbb{C} \oplus \mathbb{C} \cdots \oplus \mathbb{C}$, where we have $n$ copies of $\mathbb{C}$. We have that $A_{01}=\bigoplus_{r=1}^{m} A_{01}^{r}$, so $\sqrt{\operatorname{dim}\left(A_{01}^{r}\right)}$ is the number of paths from $A_{00}$ to $A_{01}^{r}$ on $\Gamma_{G}$, since the inclusion $A_{00} \subset A_{01}$ is given by the matrix $G$. Hence we can use the set of all paths from $A_{00}$ to $A_{01}$ as an orthonormal basis for the Hilbert space, on which $A_{01}$ acts. We work in the general setting, where the inclusion graphs are allowed multiple edges, and use the notation from [18].

Definition 2.1. Let

$$
S=\left\{(i, j, k, \rho, \sigma) \mid G_{i j} H_{j k} \neq 0,1 \leq \rho \leq G_{i j}, 1 \leq \sigma \leq H_{j k}\right\} .
$$

Then $S$ labels the paths from $A_{00}$ to $A_{11}$ via $A_{01}$, that is, $(i, j, k, \rho, \sigma)$ labels the path denoted by $\xi_{(i, j, k)}^{(\rho, \sigma)}$ on $G H$ going $A_{00}^{i}-A_{01}^{j}-A_{11}^{k}$, using the edge $\rho$ between the vertices $i$ and $j$ and the edge $\sigma$ between the vertices $j$ and $k$. These edges exist since $G_{i j} H_{j k} \neq 0$. The set $\mathcal{S}:=\left\{\xi_{(i, j, k)}^{(\rho, \sigma)} \mid(i, j, k, \rho, \sigma) \in S\right\}$ is an orthonormal basis for $\mathcal{H}:=\bigoplus_{r=1}^{q} \mathbb{C} \sqrt{\operatorname{dim}\left(A_{11}^{r}\right)}$.

\section{Definition 2.2. Let}

$$
T=\left\{(i, l, k, \zeta, \psi) \mid K_{i l} L_{l k} \neq 0,1 \leq \zeta \leq K_{i l}, 1 \leq \psi \leq L_{l k}\right\} .
$$

Then $T$ labels the paths from $A_{00}$ to $A_{11}$ via $A_{10}$, that is, $(i, l, k, \zeta, \psi)$ labels the path denoted by $\eta_{(i, l, k)}^{(\zeta, \psi)}$ on $K L$ going $A_{00}^{i}-A_{10}^{l}-A_{11}^{k}$ using the edge $\zeta$ between the vertices $i$ and $l$ and the edge $\psi$ between the vertices $l$ and $k$. These edges exist since $K_{i l} L_{l k} \neq 0$. The set $\mathcal{T}:=\left\{\eta_{(i, l, k)}^{(\zeta, \psi)} \mid(i, l, k, \zeta, \psi) \in T\right\}$ is an orthonormal basis for $\mathcal{H}=\bigoplus_{r=1}^{q} \mathbb{C} \sqrt{\operatorname{dim}\left(A_{11}^{r}\right)}$.

When $(i, j, k, \rho, \sigma) \in S$ and $(i, l, k, \zeta, \psi) \in T$ we have what is called a cell consisting of two paths, one in $S$ and one in $T$ with the same startpoint and the same endpoint. We can either use $\mathcal{S}$ or $\mathcal{T}$ as an orthonormal basis for the Hilbert space $\mathcal{H}=\bigoplus_{r=1}^{q} \mathbb{C}^{\sqrt{\operatorname{dim}\left(A_{11}^{r}\right)}}$, on which $A_{11}$ acts. So the bases $\mathcal{S}$ and $\mathcal{T}$ are unitarily equivalent, i.e. there is a unitary operator $U: \mathcal{H} \rightarrow \mathcal{H}$ identifying the bases. Let

$$
u_{(j, \rho, \sigma),(l, \zeta, \psi)}^{(i, k)}=\left\langle U \xi_{(i, j, k)}^{(\rho, \sigma)}, \eta_{(i, l, k)}^{(\zeta, \psi)}\right\rangle,
$$

with $\langle$,$\rangle denoting the inner product on the Hilbert space \mathcal{H}$. We will also sometimes denote this by $\langle\mid\rangle$ in order to avoid confusion. Then $U$ has the form

$$
U=\bigoplus_{(i, k)} u^{(i, k)}, \quad u^{(i, k)}=\left(u_{(j, \rho, \sigma),(l, \zeta, \psi)}^{(i, k)}\right)_{(i, j, k, \rho, \sigma) \in S,(i, l, k, \zeta, \psi) \in T},
$$


i.e. $U$ is a direct sum of unitary matrices. Observe that the entries of $U$ are labeled by the cells and that condition (2.2) can be restated as

$$
U \xi_{(i, j, k)}^{(\rho, \sigma)}=\sum_{l, \zeta, \psi} u_{(j, \rho, \sigma),(l, \zeta, \psi)}^{(i, k)} \eta_{(i, l, k)}^{(\zeta, \psi)},
$$

thus in the $\eta$-basis the $\xi$-basis is written as above. Note that we have used neither the commuting square nor the non-degeneracy condition, hence having such a unitary matrix $U$ is equivalent to just having an inclusion of four multi-matrix algebras.

The following theorem is due to to Ocneanu [13], [14; see also [18].

Theorem 2.3. Let $S$ and $T$ be as in Definitions 2.1 and 2.2. The square of multimatrix algebras

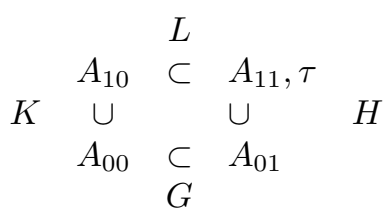

where $G H=K L$ and $\tau$ is a normalized trace on $A_{11}$, is a non-degenerate commuting square, if and only if there exists a unitary matrix $U$,

$$
U=\bigoplus_{(i, k)} u^{(i, k)}, \quad u^{(i, k)}=\left(u_{(j, \rho, \sigma),(l, \zeta, \psi)}^{(i, k)}\right)_{(i, j, k, \rho, \sigma) \in S,(i, l, k, \zeta, \psi) \in T},
$$

so that $U$ is a direct sum of unitary matrices, and such that if

$$
v_{(i, \rho, \zeta),(k, \sigma, \psi)}^{(j, l)}=\sqrt{\frac{\alpha_{i} \delta_{k}}{\theta_{j} \gamma_{l}}} \overline{u_{(j, \rho, \sigma)(l, \zeta, \rho)}^{(i, k)}}
$$

(where we use $\bar{z}$ to denote the complex conjugate of $z$ ), then

$$
V=\bigoplus_{(j, l)} v^{(j, l)}, \quad v^{(j, l)}=\left(v_{(i, \rho, \zeta),(k, \sigma, \psi)}^{(j, l)}\right)_{(i, j, k, \rho, \sigma) \in S,(i, l, k, \zeta, \psi) \in T},
$$

is also a direct sum of unitary matrices, where $\vec{\alpha}, \vec{\theta}, \vec{\gamma}$, and $\vec{\delta}$ denotes the trace vectors corresponding to $\tau$ on $A_{00}, A_{01}, A_{10}$, and $A_{11}$, respectively.

The matrix $U$ is called a connection and the condition in the theorem is called the bi-unitary condition. Note that we use here the notation in 4. The notation in [18, does not use the complex conjugation in (2.4). Thus the matrix $V$ in [18] differs from our $V$ above by a complex conjugation of the entries.

2.2. A lattice of commuting squares. Recall that for an inclusion $A \subset B$ of multi-matrix algebras, a Markov trace of modulus $\lambda(\lambda>0)$ on $B$ is the unique normalized trace $\tau_{0}$ on $B$, which has an extension to a trace $\tau_{1}$ on $B_{1}$ such that $\tau_{1}(x e)=\lambda^{-1} \tau_{0}(x)$ for all $x \in B$, where $A \subset B \subset B_{1}=\langle B, e\rangle$ is the basic construction [8]; see also [5]. Then the trace $\tau_{1}$ is a Markov trace of modulus $\lambda$ for the inclusion $B \subset B_{1}$, hence in this way we get a Markov trace on each algebra in the tower $A \subset B \subset B_{1} \subset B_{2} \subset \cdots$. Thus we get that ${\overline{\bigcup_{i=1}^{\infty} B_{i}}}^{w}$ has the unique trace $\tau$, where $\left.\tau\right|_{B_{i}}=\tau_{i}$ for all $i$, hence is the hyperfinite $\mathrm{II}_{1}$ factor. Moreover when $G$ denotes the inclusion matrix of $A \subset B$, then $\tau$ is a Markov trace of modulus $\lambda$ for $A \subset B$ if and only if $G^{t} G \vec{s}=\lambda \vec{s}$, where $\vec{s}$ is the trace vector corresponding to $\tau$, and in this case $\lambda=\|G\|^{2}=[B: A][\underline{8}],[5]$. 
Suppose from now on that the normalized trace $\tau$ on $A_{11}$ is a Markov trace for the inclusion $A_{10} \subset A_{11}$ of modulus $\|L\|^{2}$. Then since the commuting square is supposed to be non-degenerate, $\tau$ is also a Markov trace for the inclusion $A_{01} \subset A_{11}$ of modulus $\|H\|^{2}$, and its restrictions gives Markov traces for the inclusions $A_{00} \subset$ $A_{10}$ and $A_{00} \subset A_{01}$ of modulus $\|K\|^{2}$, respectively $\|G\|^{2}$. Moreover $\|K\|=\|H\|$ and $\|G\|=\|L\|[18$; see also [5], [9]. Apply the basic construction both vertically and horizontally, i.e. let $A_{12}=\left\langle A_{11}, e_{1}\right\rangle$, where $e_{1}$ is the Jones projection of $A_{11}$ onto $A_{10}$. Then

$$
A_{00} \subset A_{01} \subset\left\langle A_{01}, e_{1}\right\rangle=: A_{02}
$$

is also the basic construction, due to the non-degeneracy [9]. Similarly let $A_{21}=$ $\left\langle A_{11}, f_{1}\right\rangle$, where $f_{1}$ is the Jones projection of $A_{11}$ onto $A_{01}$. Then

$$
A_{00} \subset A_{10} \subset\left\langle A_{10}, f_{1}\right\rangle=: A_{20}
$$

is also the basic construction [9]. Iterating this process gives a lattice of commuting squares

$$
\begin{aligned}
& \begin{array}{rrrrrrrr}
\vdots & & & \vdots & & \vdots & & \\
A_{20} & \stackrel{G}{C} & A_{21} & G^{t}, e_{1} & & A_{22} & \stackrel{G}{C} & \ldots
\end{array} \\
& \begin{array}{rrrrrrrr}
K^{t}, f_{1} \cup & & H^{t}, f_{1} \cup & & K^{t}, f_{1} \cup & & \\
A_{10} & \stackrel{L}{\complement} & A_{11} & L^{t}, e_{1} & A_{12} & \stackrel{L}{\subset} & \cdots
\end{array} \\
& \begin{array}{llllllll}
K \cup & & H \cup & \multicolumn{9}{c}{K \cup} & & \\
A_{00} & \stackrel{G}{\complement} & A_{01} & G^{t}, e_{1} & A_{02} & \stackrel{G}{\complement} & \ldots
\end{array}
\end{aligned}
$$

Note that if the original commuting square has connection $U$, then the next nondegenerate commuting square which we get by applying the basic construction either vertically or horizontally has connection $V$, where $U$ and $V$ are related as in Theorem 2.3. We have

$$
A_{i j}=\left\langle A_{i, j-1}, e_{j-1}\right\rangle \text { for } j \geq 2, \quad A_{i j}=\left\langle A_{i-1, j}, f_{i-1}\right\rangle \text { for } i \geq 2,
$$

where $\left\{e_{1}, e_{2}, e_{3}, \ldots\right\}$ and $\left\{f_{1}, f_{2}, f_{3}, \ldots\right\}$ are the horizontal, respectively vertical, Jones projections. Since the trace $\tau$ on $A_{11}$ is Markov, it extends uniquely to a trace $\tau$ on each of the algebras $A_{i j}$. Let

$$
A_{i \infty}={\overline{\bigcup_{j=1}^{\infty} A_{i j}}}^{w}, \quad A_{\infty j}={\overline{\bigcup_{i=1}^{\infty} A_{i j}}}^{w}, \quad A_{\infty \infty}={\overline{\bigcup_{i, j=1}^{\infty} A_{i j}}}^{w}
$$

for all $i, j \geq 0$. Then $\tau$ extends by continuity to these algebras, and $\tau$ is the unique trace, hence we get factors, which clearly are hyperfinite. The first unital inclusion of hyperfinite factors, which we got from applying the horizontal basic construction, namely $A_{0 \infty} \subset A_{1 \infty}$, will be called the horizontal subfactor. It has index $\|K\|^{2}$ and its basic construction is given by $A_{0 \infty} \subset A_{1 \infty} \subset A_{2 \infty} \subset \cdots$. Similarly we call the first unital inclusion of hyperfinite factors, which we got from applying the vertical basic construction, namely $A_{\infty 0} \subset A_{\infty 1}$, the vertical subfactor. It has index $\|G\|^{2}$ and its basic construction is given by $A_{\infty 0} \subset A_{\infty 1} \subset A_{\infty 2} \subset \cdots$. 
2.3. The string algebra representation. In this section we introduce the string algebra representation following [13]; see also [4] and [3]. The goal is to define an automorphism of each algebra $A_{i j}$ in (2.5), and then extend it to an automorphism of both the vertical and the horizontal subfactors. In order to achieve this we will work in the string algebra representation of $A_{i j}$. Fix $i$ and $j$ and consider the algebra $A_{i j}=\bigoplus_{r=1}^{s(i, j)} A_{i j}^{r}$, where $A_{i j}^{r}$ is a matrix algebra. A basis for the Hilbert space on which $A_{i j}$ acts is given by paths of length $i+j$ (where the length of a path is the number of edges in the path) on the graphs $\Gamma_{X}$, where $X \in$ $\left\{G, H, K, L, G^{t}, H^{t}, K^{t}, L^{t}\right\}$, going $i$ steps up and $j$ steps to the right in (2.5). Thus a path starts either on $\Gamma_{K}$ or on $\Gamma_{G}$. Consider a fixed ordering of these $i$ steps up and $j$ steps to the right. This fixes a Bratteli diagram for the inclusion $A_{00} \subset A_{i j}$. An orthonormal basis for the Hilbert space on which $A_{i j}^{r}$ acts is in this representation given by all paths on this fixed Bratteli diagram ending at the vertex labeling the simple summand $A_{i j}^{r}$ of the algebra $A_{i j}$. As an example consider the inclusion $A_{00} \subset A_{22}$. The different Bratteli diagrams for this inclusion are $\Gamma_{K} \Gamma_{K^{t}} \Gamma_{G} \Gamma_{G^{t}}, \Gamma_{K} \Gamma_{L} \Gamma_{H^{t}} \Gamma_{G^{t}}, \Gamma_{K} \Gamma_{L} \Gamma_{L^{t}} \Gamma_{K^{t}}, \Gamma_{G} \Gamma_{H} \Gamma_{L^{t}} \Gamma_{K^{t}}$, $\Gamma_{G} \Gamma_{G^{t}} \Gamma_{K} \Gamma_{K^{t}}$. Moreover the dimension of $A_{i j}^{r}$ is the number of paths of length $i+j$ on the Bratteli diagram ending at the vertex labeling $A_{i j}^{r}$.

Suppose $A_{i j}^{r}$ has dimension $n_{r}^{2}$; then we have paths $\xi_{1}, \xi_{2}, \ldots, \xi_{n_{r}}$ from $A_{00}$ to $A_{i j}^{r}$. We define an inner product as follows.

Definition 2.4. Let

$$
\left\langle\xi_{i}, \xi_{j}\right\rangle=\delta_{i j},
$$

for paths $\xi_{i}$ and $\xi_{j}$ on a fixed Bratteli diagram for the inclusion $A_{00} \subset A_{i j}$ ending at the vertex labeling $A_{i j}^{r}$. Extend linearly in the first entry and conjugate linearly in the second entry. Then $\left\{\xi_{i} \mid 1 \leq i \leq n_{r}\right\}$ is an orthonormal basis for $H_{r}:=\mathbb{C}^{n_{r}}$. Furthermore let

$$
e_{i j}=\left(\xi_{i}, \xi_{j}\right) \in B\left(\mathbb{C}^{n_{r}}\right), \quad\left(\xi_{i}, \xi_{j}\right)\left(\xi_{k}\right)=\left\langle\xi_{k}, \xi_{j}\right\rangle \xi_{i},
$$

and extend by linearity to get a representation of the matrix units $e_{i j}$ of $A_{i j}^{r}$ with respect to the basis consisting of the paths $\xi_{1}, \xi_{2}, \ldots, \xi_{n_{r}}$. The pair $\left(\xi_{i}, \xi_{j}\right)$ is called a string and will often be denoted by $\sigma=\left(\sigma_{+}, \sigma_{-}\right)$.

We do the same construction as above for each simple summand of $A_{i j}$ and let paths with different endpoints belong to different Hilbert spaces $H_{r}, 1 \leq r \leq s(i, j)$, to get a representation of $A_{i j}$ on $H=\bigoplus_{r=1}^{s(i, j)} H_{r}$, with the obvious operations, since strings correspond to matrix units. Note that $A_{i j}^{r} \cdot H_{s}=\{0\}$ if $s \neq r$. This is called the string algebra representation of $A_{i j}$ associated to the fixed Bratteli diagram. We obtain in this way string algebra representations of all the algebras $A_{i j}, i, j \geq 0$.

Observe that a priori the string algebra representations obtained above depend on which Bratteli diagram we initially fix. But since the matrices $U$ and $V$ give identifications of paths, string algebra representations from different Bratteli diagrams are equivalent.

We aim to construct an automorphism of each algebra $A_{i j}$ by using certain graph automorphisms of the inclusion graphs, and then show that the automorphism is compatible with the structure of the lattice and preserves the trace, such that it extends to an automorphism of the subfactor. With this in mind we recall how the embedding and the trace is expressed in the string algebra representation. In particular we also include a short discussion of the Perron-Frobenius weights, since 
we will require that the graph automorphisms preserve these, from which it will easily follow that the algebra automorphism preserves the trace.

We have that $A_{i j} \subset A_{k l}$, whenever $i \leq k$ and $j \leq l$. Suppose the inclusion is given by the matrix $\tilde{G}$. Fix an orthonormal basis of paths for the Hilbert space on which $A_{i j}$ acts. Extend these paths with paths on $\Gamma_{\widetilde{G}}$ to paths which are an orthonormal basis of the Hilbert space on which $A_{k l}$ acts. This fixes string algebra representations of $A_{i j}$ and $A_{k l}$ and the embedding is given as follows.

Definition 2.5. Let $\sigma=\left(\sigma_{+}, \sigma_{-}\right) \in A_{i j} \subset A_{k l}$. Then

$$
\sigma=\left(\sigma_{+}, \sigma_{-}\right) \hookrightarrow \sum_{\rho \text { path on } \widetilde{G}}\left(\sigma_{+} \rho, \sigma_{-} \rho\right) \in A_{k l},
$$

where $\widetilde{G}$ is the inclusion matrix corresponding to the embedding of $A_{i j}$ into $A_{k l}$ as discussed above, and where it is to be understood that the starting point of $\rho$ is the endpoint of $\sigma$ such that $\sigma_{+} \rho$ and $\sigma_{-} \rho$ are paths obtained by concatenation in the obvious way.

Let $\beta=\|G\|=\|L\|$ and $\beta^{\prime}=\|K\|=\|H\|$. Then the trace vector $\vec{\delta}=$ $\left(\delta_{1}, \ldots, \delta_{q}\right)$ on the algebra $A_{11}$ is the Perron-Frobenius eigenvector of $L^{t} L$ (i.e. $L^{t} L \vec{\delta}$ $\left.=\beta^{2} \vec{\delta}\right)$ normalized such that $\sum_{r=1}^{q} \delta_{r} \sqrt{\operatorname{dim}\left(A_{11}^{r}\right)}=1$, where $A_{11}^{r}$ denotes the $r$ th simple summand of $A_{11}$, since $\tau$ is the Markov trace of modulus $\beta^{2}$ for the inclusion $A_{10} \subset A_{11}$. Since $\tau$ is a Markov trace of modulus $\left(\beta^{\prime}\right)^{2}$ for the inclusion $A_{01} \subset A_{11}$, we also have that $H^{t} H \vec{\delta}=\left(\beta^{\prime}\right)^{2} \vec{\delta}$. We denote the trace vectors on the algebras $A_{00}, A_{01}$, and $A_{10}$, by $\vec{\alpha}, \vec{\theta}$, and $\vec{\gamma}$, respectively. Let $\xi_{1}=\left(\beta^{\prime} \vec{\gamma}, \beta^{\prime} \beta \vec{\delta}\right)$. Then $\Delta_{L} \xi_{1}=\beta \xi_{1}$, hence

$$
\begin{aligned}
\beta \xi_{1}(i) & =\left(\Delta_{L} \xi_{1}\right)(i)=\sum_{j=1}^{q+p}\left(\Delta_{L}\right)_{i j} \xi_{1}(j) \\
& =\sum_{j=1}^{q+p}\left(\text { number of edges from } i \text { to } j \text { in } \Gamma_{L}\right) \xi_{1}(j),
\end{aligned}
$$

where $i$ and $j$ are vertices of $\Gamma_{L}$, and where the even vertices of $\Gamma_{L}$ label the direct summands of $A_{10}$ and the odd vertices label the direct summands of $A_{11}$. The entries of the vector $\xi_{1}$ are called the Perron-Frobenius weights of the corresponding vertices of $\Gamma_{L}$. Similarly let $\xi_{2}=\left(\beta \vec{\theta}, \beta \beta^{\prime} \vec{\delta}\right)$ to obtain

$$
\begin{aligned}
\beta^{\prime} \xi_{2}(i) & =\left(\Delta_{H} \xi_{2}\right)(i)=\sum_{j=1}^{q+m}\left(\Delta_{H}\right)_{i j} \xi_{2}(j) \\
& \left.=\sum_{j=1}^{q+m} \text { (number of edges from } i \text { to } j \text { in } \Gamma_{H}\right) \xi_{2}(j),
\end{aligned}
$$

where $i$ and $j$ are vertices of $\Gamma_{H}$, and where the even vertices of $\Gamma_{H}$ label the direct summands of $A_{01}$ and the odd vertices label the direct summands of $A_{11}$. Finally by using either $\xi_{3}=(\vec{\alpha}, \beta \vec{\theta})$ or $\xi_{4}=\left(\vec{\alpha}, \beta^{\prime} \vec{\gamma}\right)$, we get the Perron-Frobenius weights of the vertices labeling direct summands of $A_{00}$ and either $A_{01}$ or $A_{10}$. Simply use $\xi$ to denote any of the Perron-Frobenius vectors above and $\xi(i)$ to denote the PerronFrobenius weight of the vertex $i$. Then by applying equations (2.6) and (2.7) we 
obtain the following useful identity. Let $i$ and $j$ be fixed and let $\sigma=\left(\sigma_{+}, \sigma_{-}\right) \in A_{i j}$. Then for any $k$ and $l$ with $A_{i j} \underset{\widetilde{G}}{\subsetneq} A_{k l}$, we get

$$
\xi(r(\sigma))=\sum_{\alpha \text { path on } \widetilde{G}, s(\alpha)=r(\sigma)}\left(\beta^{\prime}\right)^{-(k-i)} \beta^{-(l-j)} \xi(r(\alpha)),
$$

where $r(\sigma)$ and $s(\sigma)$ denotes the end point, respectively the starting point, of the string $\sigma$.

Consider the algebras $A_{i j}$ from (2.5). Then it follows from (2.8) that we can define the normalized trace, which comes from the Markov trace and is compatible with embeddings as follows. See for instance [4].

Lemma 2.6. Let $\sigma=\left(\sigma_{+}, \sigma_{-}\right) \in A_{i j}$ for some $i, j \geq 0$. Then

$$
\tau(\sigma)=\tau\left(\left(\sigma_{+}, \sigma_{-}\right)\right)=\delta_{\sigma_{+}, \sigma_{-}}\left(\beta^{\prime}\right)^{-i} \beta^{-j} \xi(r(\sigma))
$$

extended by linearity is a normalized trace on $A_{i j}$ compatible with embeddings.

Note that the trace does not depend on the representation of the algebra $A_{i j}$.

2.4. Construction of subfactor automorphisms. We aim to construct an automorphism of a commuting square of finite-dimensional $\mathrm{C}^{*}$-algebras, and use this to construct a subfactor automorphism.

Definition 2.7. Consider the commuting square (2.1). A map $\varphi \in \operatorname{Aut}\left(A_{11}\right)$ is called an automorphism of the commuting square or a commuting square automorphism, if the following are satisfied:

- $\tau \circ \varphi=\tau$,

- $\varphi\left(A_{00}\right)=A_{00}, \varphi\left(A_{01}\right)=A_{01}, \varphi\left(A_{10}\right)=A_{10}$.

It is well known that an automorphism $\varphi \in \operatorname{Aut}\left(A_{11}\right)$ of a commuting square can be extended iteratively to an automorphism of each commuting square in the lattice of commuting squares, which we get by iterating the basic construction, by letting $\phi\left(e_{i}\right)=e_{i}$ and $\phi\left(f_{i}\right)=f_{i}$. See for instance [12.

Recall that a graph automorphism is a bijection on the graph, which maps vertices to vertices, and adjacent edges to adjacent edges. Let $\phi$ be a collection of graph automorphisms, one for each of the four bipartite graphs $\Gamma_{G}, \Gamma_{L}, \Gamma_{K}$, and $\Gamma_{H}$, which preserves Perron-Frobenius weights (i.e. $\phi$ maps a vertex to a vertex which has the same Perron-Frobenius weight), and agrees on the vertices that are identified in the commuting square as explained previously. Then in particular $\phi$ is a bijection on the set of vertices labeled by $\left(\Gamma_{X}\right)_{\text {odd }}$ or $\left(\Gamma_{X}\right)_{\text {even }}$, where $X$ is any of $G, K, L$, or $H$, and $\phi$ is also a bijection on the set of edges of each graph $\Gamma_{G}, \Gamma_{K}, \Gamma_{L}$, and $\Gamma_{H}$. We would like to use the map $\phi$ to define an automorphism $\varphi$ of each algebra $A_{i j}$ using the string algebra representation. Given a fixed Bratteli diagram for the inclusion $A_{00} \subset A_{i j}$, we can write a path as an alternating sequence of $i+j+1$ vertices and $i+j$ edges, say $\xi=\left(z_{0}, \epsilon_{1}, z_{1}, \epsilon_{2}, \ldots, \epsilon_{i+j}, z_{i+j}\right)$, where $z_{i}$ denotes a vertex and $\epsilon_{i}$ denotes an edge between the vertices $z_{i-1}$ and $z_{i}$.

Definition 2.8. Let $i, j \geq 0$. Let $\phi$ be a collection of graph automorphisms as defined above. We extend $\phi$ to a map on paths also denoted by $\phi$ as follows. For a path $\xi=\left(z_{0}, \epsilon_{1}, z_{1}, \epsilon_{2}, \ldots, \epsilon_{i+j}, z_{i+j}\right)$ on a fixed Bratteli diagram for $A_{00} \subset A_{i j}$, we let

$$
\phi(\xi)=\left(\phi\left(z_{0}\right), \phi\left(\epsilon_{1}\right), \phi\left(z_{1}\right), \phi\left(\epsilon_{2}\right), \ldots, \phi\left(\epsilon_{i+j}\right), \phi\left(z_{i+j}\right)\right)
$$


and extend by linearity. Then $\phi$ is a bijection on the sets of paths on any fixed Bratteli diagram in (2.5), which are the basis for the fixed string algebra representation of $A_{i j}$.

For a string $\sigma=\left(\sigma_{+}, \sigma_{-}\right) \in A_{i j}$ we define

$$
\varphi_{i j}(\sigma)=\left(\phi\left(\sigma_{+}\right), \phi\left(\sigma_{-}\right)\right) .
$$

By linearity we extend this definition to all of $A_{i j}$, hence we get a map $\varphi_{i j}$ of the algebra $A_{i j}$ in the string algebra representation defined by the fixed Bratteli diagram for $A_{00} \subset A_{i j}$.

Consider a fixed string algebra representation of the algebra $A_{i j}$. Since $\phi$ is a bijection of the orthonormal basis of the Hilbert space on which $A_{i j}$ acts, we have that $\phi$ is just a basis change, i.e. given by some unitary, say $w_{\phi}$. Hence $\varphi_{i j}$ is given by $\operatorname{Ad} w_{\phi}$. By using the definition of the map $\varphi_{i j}$ and the string algebra formalism we easily obtain the following result.

Lemma 2.9. Consider a fixed string algebra representation of the algebra $A_{i j}$ for some $i, j \geq 0$. Then the map $\varphi_{i j}$ as defined above is a ${ }^{*}$-homomorphism of $A_{i j}$.

Our goal is to find a condition on the connection such that $\varphi_{11}$ is an automorphism of the commuting square (2.1). Consider $A_{11}$. Recall that $\xi_{(i, j, k)}^{(\rho, \sigma)}$ is a path on $G H, \eta_{(i, l, k)}^{(\zeta, \psi)}$ is a path on $K L$, and

$$
\xi_{(i, j, k)}^{(\rho, \sigma)}=\sum_{l, \zeta, \psi} u_{(j, \rho, \sigma),(l, \zeta, \psi)}^{(i, k)} \eta_{(i, l, k)}^{(\zeta, \psi)}
$$

In order for $\varphi_{11}$ to be a commuting square automorphism, we must show that the definition of $\varphi_{11}$ is independent of the basis in which we represent $A_{11}$. By rewriting the paths $\xi$ and $\eta$ using sequences of alternating vertices and edges as above we get that $\xi_{(i, j, k)}^{(\rho, \sigma)}=(i, \rho, j, \sigma, k)$ and $\eta_{(i, l, k)}^{(\zeta, \psi)}=(i, \zeta, l, \psi, k)$, hence

$$
\varphi\left(\xi_{(i, j, k)}^{(\rho, \sigma)}\right)=(\varphi(i), \varphi(\rho), \varphi(j), \varphi(\sigma), \varphi(k))=\xi_{(\phi(i), \phi(j), \phi(k))}^{(\phi(\rho), \phi(\sigma))}
$$

and

$$
\varphi\left(\eta_{(i, l, k)}^{(\zeta, \psi)}\right)=(\varphi(i), \varphi(\zeta), \varphi(l), \varphi(\psi), \varphi(k))=\eta_{(\phi(i), \phi(l), \phi(k))}^{(\phi(\zeta), \phi(\psi))} .
$$

This means that on the one hand we have

$$
\begin{aligned}
\phi\left(\xi_{(i, j, k)}^{(\rho, \sigma)}\right) & =\phi\left(\sum_{l, \zeta, \psi} u_{(j, \rho, \sigma),(l, \zeta, \psi)}^{(i, k)} \eta_{(i, l, k)}^{(\zeta, \psi)}\right)=\sum_{l, \zeta, \psi} u_{(j, \rho, \sigma),(l, \zeta, \psi)}^{(i, k)} \phi\left(\eta_{(i, l, k)}^{(\zeta, \psi)}\right) \\
& =\sum_{l, \zeta, \psi} u_{(j, \rho, \sigma),(l, \zeta, \psi)}^{(i, k)} \eta_{(\phi(i), \phi(l), \phi(k))}^{(\phi(\zeta), \phi(\psi))},
\end{aligned}
$$

and on the other hand we have

$$
\begin{aligned}
\phi\left(\xi_{(i, j, k)}^{(\rho, \sigma)}\right) & =\xi_{(\phi(i), \phi(j), \phi(k))}^{(\phi(\rho), \phi(\sigma))}=\sum_{l, \zeta, \psi} u_{(\phi(j), \phi(\rho), \phi(\sigma)),(l, \zeta, \psi)}^{(\phi(i), \phi(k))} \eta_{(\phi(i), l, \phi(k))}^{(\zeta, \psi)} \\
& =\sum_{l, \zeta, \psi} u_{(\phi(j), \phi(\rho), \phi(\sigma)),(\phi(l), \phi(\zeta), \phi(\psi))}^{(\phi(i), \phi(k))} \eta_{(\phi(i), \phi(l), \phi(k))}^{(\phi(\zeta), \phi(\psi))}
\end{aligned}
$$

where we have used that $\phi$ is a bijection on the set of vertices and on the set of edges. This means that in order to get an automorphism $\varphi_{11}$ of the commuting square (2.1) the following must be satisfied; see for instance [6], [10]. 
Definition 2.10. Let $\phi$ be as above. Let $U$ be the connection of (2.1). If

$$
u_{(j, \rho, \sigma),(l, \zeta, \psi)}^{(i, k)}=u_{(\phi(j), \phi(\rho), \phi(\sigma)),(\phi(l), \phi(\zeta), \phi(\psi))}^{(\phi(i), \phi(k))}
$$

for all $(i, j, k, \rho, \sigma) \in S$ and for all $(i, l, k, \zeta, \psi) \in T$, then we say that the connection is symmetric with respect to $\phi$, or that it is symmetric.

We know that $\phi$ maps paths to paths, since it is defined in Definition 2.8 from a collection of graph automorphisms of all the four inclusion graphs in the commuting square. Since the entries of the connection $U$ are labeled by cells, equation (2.11) says that the unitary matrix $U$, which gives the connection, is invariant under the map $\phi$. By this we mean that the value of the entry of the connection $U$ labeled by any given cell is the same as the value of the entry of the connection $U$ labeled by the new cell, which we get by applying $\phi$ to the paths in the given cell; see (2.11). Due to the relationship between the matrices $U$ and $V$ this implies that $V$ is also invariant under the map $\phi$. Assume from now on that the connection $U$ of the commuting square (2.1) is symmetric with respect to $\phi$.

Lemma 2.11. Let $i, j \geq 0$ be fixed. The definition of the map $\varphi_{i j}$ on the algebra $A_{i j}$ does not depend on the string algebra representation of $A_{i j}$.

Proof. This follows by induction using the fact that both $U$ and $V$ are invariant under $\phi$.

Lemma 2.12. Let $i, j \geq 0$ be fixed. We have that $\left.\varphi_{i j}\right|_{A_{r s}}=\varphi_{r s}$ for all $r \leq i$ and $s \leq j$.

Proof. It is enough to show that $\left.\varphi_{i j}\right|_{A_{r s}}=\varphi_{r s}$ for $r=i-1$ and $s=j$, and for $r=i$ and $s=j-1$. The cases are identical and are proved using the fact that $\phi$ is a bijection.

Lemma 2.13. The automorphism $\varphi_{i j}$ is trace preserving.

Proof. Easy computation using the fact that $\phi$ is a bijection on the set of paths and preserves Perron-Frobenius weights.

We have now proved the following proposition, which is well known to experts.

Proposition 2.14. The map $\varphi_{11}$ is an automorphism of the commuting square (2.1), where $\tau$ is the Markov trace of modulus $\|L\|^{2}$ for the inclusion $A_{10} \subset A_{11}$.

Notice that in general there may be automorphisms of a commuting square of finite-dimensional $C^{*}$-algebras, which do not arise in this way, i.e. via graph symmetries. The automorphism of the commuting square from a graph symmetry corresponds to permutations of the paths, but in general other unitaries may be allowed.

Definition 2.15. Let $N \subset M$ be a subfactor of type $\mathrm{II}_{1}$. The subfactor automorphisms of $N \subset M$ is the group $\operatorname{Aut}(M, N)=\{\theta \in \operatorname{Aut}(M) \mid \theta(N)=N\}$. The inner automorphisms is the $\operatorname{group} \operatorname{Int}(M, N)=\{\operatorname{Ad} u \mid u \in \mathcal{U}(N)\}$.

Definition 2.16. Consider the commuting square (2.1) with connection $U$, which is symmetric with respect to the map $\phi$. Let $\varphi_{i j}$ be the automorphisms of the algebras $A_{i j}$ as in Definition 2.8. Define the map $\varphi$ on $\bigcup_{i, j=0}^{\infty} A_{i j}$ by $\varphi(\sigma)=\varphi_{i j}(\sigma)$ for $i, j$ such that $\sigma \in A_{i j}$. Since $\varphi$ is trace preserving it extends to $A_{\infty, \infty}$ by continuity. Thus we get an automorphism in $\operatorname{Aut}\left(A_{1, \infty}, A_{0, \infty}\right)$ and an automorphism 
in $\operatorname{Aut}\left(A_{\infty, 0}, A_{\infty, 1}\right)$, i.e. an automorphism of both the vertical and the horizontal subfactors.

As noted earlier we could also have extended the map $\varphi_{11}$ to the full lattice of commuting squares (2.5) by letting it leave the Jones projections invariant. In fact it can be shown that the map $\varphi$ defined above does leave the Jones projections invariant, hence extending the map $\varphi_{11}$ by letting it leave the Jones projections invariant gives the same automorphism. When $\varphi$ switches some of the simple summands of some of the algebras $A_{i j}$, it follows that $\varphi$ cannot be an inner automorphism. Let us summarize the above discussion in the following.

Theorem 2.17. Consider the non-degenerate commuting square

$$
\begin{aligned}
& \text { L } \\
& K \begin{array}{cccc}
A_{10} & \subset & A_{11}, \tau & \\
\cup & & \cup & \cup \\
A_{00} & \subset & A_{01} &
\end{array}
\end{aligned}
$$

where $\tau$ is the Markov trace. Let $\phi$ be a collection of graph automorphism of all the four bipartite graphs $\Gamma_{G}, \Gamma_{L}, \Gamma_{K}$, and $\Gamma_{H}$, which preserves Perron-Frobenius weights, and agrees on the vertices that are identified in the commuting square. Suppose that $\phi$ is non-trivial on the set of vertices, and suppose that the commuting square has a symmetric connection with respect to $\phi$. Let $N \subset M$ denote either the vertical or the horizontal subfactor. Define the map $\varphi \in \operatorname{Aut}(M, N)$ as in Definition 2.16. Then $\varphi$ is not an inner automorphism, i.e. $\varphi$ is not of the form $\operatorname{Ad}(u)$ for some $u \in \mathcal{U}(N)$.

Proof. We will show that $\varphi \in \operatorname{Out}(M, N)=\operatorname{Aut}(M, N) / \operatorname{Int}(M, N)$, where $N \subset M$ denotes the horizontal subfactor. The proof that $\varphi \in \operatorname{Out}(M, N)$, where $N \subset M$ is the vertical subfactor is identical. Suppose $\varphi$ is inner, i.e. $\varphi=\operatorname{Ad} u$ for some $u \in \mathcal{U}(N)$. We have the sequence of commuting squares:

$$
\begin{array}{cccccccccccc}
A_{10} & \subset & A_{11} & \subset & A_{12} & \subset & \cdots & \subset & A_{1, \infty} & = & M \\
\cup & & \cup & & \cup & & & & \cup & & \cup \\
A_{00} & \subset & A_{01} & \subset & A_{02} & \subset & \cdots & \subset & A_{0, \infty} & = & N
\end{array}
$$

In particular for each $n \in \mathbb{N}$

$$
\begin{array}{ccc}
A_{1 n} & \subset & M \\
\cup & & \cup \\
A_{0 n} & \subset & N
\end{array}
$$

is a commuting square. For all $x \in A_{1 n}$ we have $\varphi(x) u=u x$. Now apply the conditional expectation $E_{A_{1 n}}: M \mapsto A_{1 n}$; then

$$
E_{A_{1 n}}(u) x=E_{A_{1 n}}(u x)=E_{A_{1 n}}(\varphi(x) u)=\varphi(x) E_{A_{1 n}}(u),
$$

since $x$ and $\varphi(x)$ are in $A_{1 n}$. Let $w_{n}=E_{A_{1 n}}(u)$. Then $w_{n} \in A_{0 n}$, since (2.13) is a commuting square and the above equation reads

$$
w_{n} x=\varphi(x) w_{n} .
$$

Furthermore $\lim _{n \rightarrow \infty}\left\|E_{A_{1 n}}(u)-u\right\|_{2}=0$, hence also $\lim _{n \rightarrow \infty}\left\|1-w_{n}^{*} w_{n}\right\|_{2}=0$, thus the support projection of $w_{n}^{*} w_{n}$ converges to 1 . 
Since $\phi$ is non-trivial on the set of vertices, we have that $\phi$ is either non-trivial on the set of vertices of $\Gamma_{G}$ or non-trivial on the set of vertices of $\Gamma_{L}$. Suppose that $\phi$ is non-trivial on the set of vertices of $\left(\Gamma_{L}\right)_{\text {even }}$. The case where $\phi$ is non-trivial on the set of vertices of $\left(\Gamma_{L}\right)_{\text {odd }}$ is similar. Let $n$ be even. Then $A_{1 n}=\bigoplus_{i=1}^{r} A_{1 n}^{i}$, where $A_{1 n}^{i}$ denotes the simple summands of $A_{1 n}$, and where $r$ is the number of vertices of $\left(\Gamma_{L}\right)_{\text {even }}$. The simple summands of $A_{1 n}$ are labeled by the vertices, on which $\phi$ is non-trivial, so we can assume that $\phi$ switches the vertices $k$ and $l$ for $1 \leq k, l \leq r$. Since $w_{n} \in A_{1 n}$, we have for $n$ even that $w_{n}=\bigoplus_{i=1}^{r} w_{n}^{i}$, where $w_{n}^{i} \in A_{1 n}^{i}$. Choose $n \in \mathbb{N}, n$ even such that $w_{n}^{k} \neq 0$ and $w_{n}^{l} \neq 0$. Let $x \in A_{1 n}^{k}, x \neq 0$; then $\varphi(x) \in A_{1 n}^{l}$, which is a contradiction to (2.14). The case where $\phi$ is non-trivial on the set of vertices of $\Gamma_{G}$ is shown as above by using $A_{0 n}$ instead of $A_{1 n}$.

\section{THE SERIES OF EXAMPLES}

We consider 3-star graphs, which are graphs with one triple point and where the rest of the vertices have valency two or one. The 3-star graphs we consider have two of the rays with the same length. Let $\Gamma_{k}$ denote the graph depicted in Figure 2 .

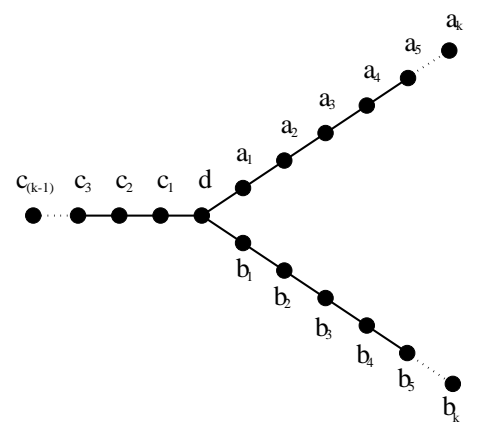

FIGURE 2 .

We consider the infinite series where $k \geq 3$ and work with the following inclusions of four multi-matrix algebras:

$$
\begin{array}{ccccc} 
& & G_{k} & & \\
G_{k} G_{k}^{t}-I & A_{10} & \subset & A_{11} & \\
& \cup & & \cup & G_{k}^{t} G_{k}-I \\
& A_{00} & \subset & A_{01} & \\
& & G_{k} & &
\end{array}
$$

where $G_{k}$ is the inclusion matrix corresponding to $\Gamma_{k}$, and $A_{00}$ is abelian. If this is a commuting square with respect to a fixed normalized trace $\tau$ on $A_{11}$, then it is clearly non-degenerate.

The graph $\Gamma_{k}$ has an obvious graph automorphism $\phi$, which switches the $a$-ray and the $b$-ray. Namely, we let $\phi(d)=d, \phi\left(a_{i}\right)=b_{i}, \phi\left(b_{i}\right)=a_{i}$ for $1 \leq i \leq k$, and $\phi\left(c_{i}\right)=c_{i}$ for $1 \leq i \leq m$. This gives a bijection on the set of vertices of $\Gamma_{k}$. Let $a_{0}=b_{0}=c_{0}=d$. Let $\phi$ map the edge $a_{i}-a_{i+1}$ to the edge $b_{i}-b_{i+1}$ and the edge $b_{i}-b_{i+1}$ to the edge $a_{i}-a_{i+1}$ for all $0 \leq i \leq k-1$. Moreover let $\phi$ map the edge $c_{i}-c_{i+1}$ to itself for all $0 \leq i \leq m-1$. Then $\phi$ is also a bijection on the set of edges and $\phi$ maps adjacent edges to adjacent edges, thus $\phi$ is a graph automorphism. 
We want to extend $\phi$ to a collection of graph automorphisms of the four bipartite graphs corresponding to the inclusion matrices in (3.1), which agrees on the vertices that are identified in the commuting square, and which preserves Perron-Frobenius weights, as in section 2.4 This determines $\phi$ except on the double edge in $G_{k} G_{k}^{t}-I$. The goal is to find a symmetric connection $U$ of (3.1) with respect to $\phi$ defined as above for the series of examples that we consider.

Commuting squares of the type above were constructed by Haagerup and Schou in 18, where many other similar examples are also considered. We use the notation of [18], and the computations we do to find a symmetric connection will be similar to the ones done by Haagerup and Schou. But our computations will be more specialized since we want to construct a symmetric connection, which means that the entries of the connection have to satisfy some additional identities besides the ones coming from the bi-unitary condition. We will construct such a connection explicitly for the infinite series of examples.

3.1. The symmetric connection. We aim to construct a connection of the commuting square (3.1) for the infinite series of the Haagerup-Schou subfactors, which is symmetric with respect to the map $\phi$ defined above. For this we need to explicitly compute two unitary matrices $U$ and $V$, which are related by a multiplication of the entries by certain coefficients, a conjugation of the entries, and a relabeling of the entries, as in Theorem 2.3. Notice that instead of computing the matrix $V$ from Theorem[2.3] we will compute the matrix $\bar{V}$, where all the entries of the matrix are conjugated, since this simplifies the notation.

The main steps of the construction are as follows. First we show that the coefficients can be easily computed using only the Perron-Frobenius weights of the graph $\Gamma_{k}$, which can be defined recursively, and are preserved by our graph automorphism. Then we describe a way to systematize the set-up by using a table with both rows and columns labeled by the edges of $\Gamma_{k}$. We label the entries of the table, which correspond to cells, i.e. to entries of the connection. This is done by taking the symmetry of the connection into account, since this greatly reduces the number of labels. The structures of the matrices $U$ and $V$ can be read off from the table, and when we compute the coefficients, we get an inverse symmetry across the diagonal. Then we start to determine the absolute values of the entries of $U$. By taking advantage of the symmetry, we only get two different $3 \times 3$ blocks, which we need to deal with. When we have determined the absolute values of the entries of all the $3 \times 3$ blocks, we determine the absolute values of the entries of all the $2 \times 2$ blocks by working our way down along the diagonal in the table. Again we can take advantage of the symmetry and only work with the ones from $U$. After having determined the absolute values of all the entries this way, we show that they are consistent. We determined absolute values of elements, which were part of either a $3 \times 3$ block of $U$ or of $V$ or part of a $2 \times 2$ block of $U$ or of $V$. But elements which are $1 \times 1$ blocks also need to be unitary. We show how this gives rise to a set of identities for the Perron-Frobenius weights, and show that these identities are satisfied. Finally we find the complex phases. This is again done by using the symmetry of the situation, in particular by assuming that the complex phases have symmetry across the diagonal in our table. First we find the complex phases for the entries of the two $3 \times 3$ blocks, and then for the entries of the $2 \times 2$ blocks by working our way down the diagonal. 
3.1.1. Set-up and structure of the connection. In order to find a symmetric connection, we first need to know the coefficients relating the matrices $U$ and $V$, and also the entries and the structure of the matrices. Let us first see how to compute the coefficients. Let $\Gamma_{k}^{0}$ denote the vertices of $\Gamma_{k}$. Then $\Gamma_{k}^{0}=\Gamma_{k}^{\text {even }} \cup \Gamma_{k}^{\text {odd }}$, where $d$ is even and

$$
\Gamma_{k}^{\text {even }}=\left\{p \in \Gamma_{k}^{0} \mid \operatorname{dist}(p, d) \text { is even }\right\}, \quad \Gamma_{k}^{\text {odd }}=\left\{p \in \Gamma_{k}^{0} \mid \operatorname{dist}(p, d) \text { is odd }\right\},
$$

with $\operatorname{dist}(p, d)$ the minimal number of edges in a path on $\Gamma_{k}$ from $d$ to $p$. Then

$$
\Gamma_{k}^{\text {even }}=\left\{d, a_{2}, b_{2}, c_{2}, a_{4}, b_{4}, c_{4}, \ldots\right\}, \quad \Gamma_{k}^{\text {odd }}=\left\{a_{1}, b_{1}, c_{1}, a_{3}, b_{3}, c_{3}, \ldots\right\} .
$$

Let $\lambda_{k}=\left\|G_{k}\right\|$ and let $\xi$ be the Perron-Frobenius eigenvector of $\Delta_{\Gamma_{k}}$ with $\xi(d)=1$. We have that $\xi=\left(\xi_{\text {even }}, \xi_{\text {odd }}\right)$, where $\xi_{\text {even }}$, respectively $\xi_{\text {odd }}$, gives the PerronFrobenius weights of the vertices in $\Gamma_{k}^{\text {even }}$, respectively $\Gamma_{k}^{\text {odd }}$. The equation

$$
\Delta_{\Gamma_{k}} \xi=\lambda_{k} \xi
$$

is referred to as the eigenvalue equation. Note that since there are no multiple edges in $\Gamma_{k}$ it can also be written as

$$
\lambda_{k} \xi(z)=\left(\Delta_{\Gamma_{k}} \xi\right)(z)=\sum_{y \text { connected to } z \in \Gamma_{k}} \xi(y),
$$

where $z$ is a vertex of $\Gamma_{k}$. It allows us to define the Perron-Frobenius weights recursively as follows [18. Define the following polynomials

$$
R_{0}(x)=1, \quad R_{1}(x)=x, \quad R_{n}(x)=x R_{n-1}(x)-R_{n-2}(x) .
$$

Then the Perron-Frobenius weights of $\Gamma_{k}$ are given by

$$
\begin{array}{rlrl}
\xi(d)=1, & \xi\left(a_{i}\right)=\xi\left(b_{i}\right)=\frac{R_{k-i}\left(\lambda_{k}\right)}{R_{k}\left(\lambda_{k}\right)} & 1 \leq i \leq k, \\
\xi\left(c_{i}\right)=\frac{R_{k-1-i}\left(\lambda_{k}\right)}{R_{k-1}\left(\lambda_{k}\right)} & 1 \leq i \leq k-1,
\end{array}
$$

where $k$ and $k-1$ are the lengths of the rays of $\Gamma_{k}$ (see Figure 2). Note that $\phi$ clearly preserves Perron-Frobenius weights since it interchanges the $a$-ray and $b$-ray of $\Gamma_{k}$. For the polynomials defined above we have the following property [18], which will be needed later.

Lemma 3.1. For any $x \geq 2$ the function $R_{n}(x)$ is an increasing function of $n \in \mathbb{N}$, whereas the function $\frac{R_{n}(x)}{R_{n+m}(x)}$ is a decreasing function of $m \in \mathbb{N}$.

We let $\vec{\delta}=\left.\eta \xi\right|_{\Gamma_{k}^{\text {odd }}}$ and choose $\eta>0$ such that $\sum_{r=1}^{q} \delta_{r} \sqrt{\operatorname{dim}\left(A_{11}^{r}\right)}=1$. Then $\vec{\delta}$ is the normalized trace vector on $A_{11}=\bigoplus_{i=1}^{r} A_{11}^{r}$ defining the Markov trace for the inclusion $A_{10} \subset A_{11}$, hence is also Markov for the inclusion $A_{01} \subset A_{11}$, and by restriction for the inclusions $A_{00} \subset A_{01}$ and $A_{00} \subset A_{10}$ too, since the commuting square is non-degenerate. Since

$$
\lambda_{k}\left(\begin{array}{c}
\xi_{\text {even }} \\
\xi_{\text {odd }}
\end{array}\right)=\lambda_{k} \xi=\Delta_{\Gamma_{k}} \xi=\left(\begin{array}{cc}
0 & G_{k} \\
G_{k}^{t} & 0
\end{array}\right)\left(\begin{array}{c}
\xi_{\text {even }} \\
\xi_{\text {odd }}
\end{array}\right)=\left(\begin{array}{c}
G_{k} \xi_{\text {odd }} \\
G_{k}^{t} \xi_{\text {even }}
\end{array}\right),
$$


we have

$$
\begin{aligned}
\vec{\gamma} & =G_{k} \vec{\delta}=G_{k} \eta \xi_{\text {odd }}=\lambda_{k} \eta \xi_{\text {even }} \\
\vec{\theta} & =\left(G_{k}^{t} G_{k}-I\right) \vec{\delta}=\left(G_{k}^{t} G_{k}-I\right) \eta \xi_{\text {odd }} \\
& =\lambda_{k} G_{k}^{t} \eta \xi_{\text {even }}-\eta \xi_{\text {odd }}=\left(\lambda_{k}^{2}-1\right) \eta \xi_{\text {odd }} \\
\vec{\alpha} & =\left(G_{k} G_{k}^{t}-I\right) G_{k} \vec{\delta}=\left(G_{k} G_{k}^{t}-I\right) G_{k} \eta \xi_{\text {odd }} \\
& =\left(G_{k} G_{k}^{t}-I\right) \lambda_{k} \eta \xi_{\text {even }}=\left(\lambda_{k}^{3}-\lambda_{k}\right) \eta \xi_{\text {even }}
\end{aligned}
$$

where $\vec{\alpha}, \vec{\theta}$, and $\vec{\gamma}$ are the trace vectors on the algebras $A_{00}, A_{01}$, and $A_{10}$, respectively. Thus for the coefficients in equation (2.4) we have

$$
\sqrt{\frac{\alpha_{\tilde{i}} \delta_{\widetilde{k}}}{\beta_{\tilde{j}} \gamma_{\tilde{l}}}}=\sqrt{\frac{\left(\lambda_{k}^{3}-\lambda_{k}\right) \eta \xi_{i} \eta \xi_{k}}{\left(\lambda_{k}^{2}-1\right) \eta \xi_{j} \lambda_{k} \eta \xi_{l}}}=\sqrt{\frac{\xi_{i} \xi_{k}}{\xi_{j} \xi_{l}}},
$$

where $\alpha_{\tilde{i}}$ corresponds to the same vertex in $A_{00}$ as $\xi_{i}$, etc.

Now we will find the cells, since these label the entries of the connection. The entries of $G_{k} G_{k}^{t}-I$ as a $\Gamma_{k}^{\text {even }} \times \Gamma_{k}^{\text {even }}$ matrix are given by

$$
\left(G_{k} G_{k}^{t}-I\right)_{p q}= \begin{cases}1, & \text { if } \operatorname{dist}(p, q)=2, \\ 0, & \text { else }\end{cases}
$$

for $p, q$ even vertices, $p \neq q$, and

$$
\left(G_{k} G_{k}^{t}-I\right)_{p p}= \begin{cases}2, & \text { if } p=d \\ 0, & \text { if } p \text { is an endpoint of } \Gamma_{k}, \\ 1, & \text { else }\end{cases}
$$

for $p$ an even vertex. The entries of $G_{k}^{t} G_{k}-I$ as a $\Gamma_{k}^{\text {odd }} \times \Gamma_{k}^{\text {odd }}$ matrix are given by

$$
\left(G_{k}^{t} G_{k}-I\right)_{p q}= \begin{cases}1, & \text { if } \operatorname{dist}(p, q)=2 \\ 0, & \text { else }\end{cases}
$$

for $p, q$ odd vertices, $p \neq q$, and

$$
\left(G_{k}^{t} G_{k}-I\right)_{p p}= \begin{cases}0, & \text { if } p \text { is an endpoint of } \Gamma_{k}, \\ 1, & \text { else }\end{cases}
$$

for $p$ an even vertex. In order to find the cells we order the edges of $\Gamma_{k}$ as $d a_{1}, d b_{1}, d c_{1}, a_{2} a_{1}, b_{2} b_{1}, c_{2} c_{1}, a_{2} a_{3}, \ldots$, and we label both columns and rows of a table by the edges of $\Gamma_{k}$, since both the horizontal inclusions $A_{00} \subset A_{01}$ and $A_{10} \subset A_{11}$ are given by the inclusion matrix $G_{k}$. Then an entry in the table corresponds to a cell whenever the left vertices of the edges are connected in $G_{k} G_{k}^{t}-I$, and the right vertices of the edges are connected in $G_{k}^{t} G_{k}-I$. This means for instance that the entry $\left(b_{2} b_{1}, d c_{1}\right)$ corresponds to a cell, since $b_{2}$ and $d$ are connected in $G_{k} G_{k}^{t}-I$, and $b_{1}$ and $c_{1}$ are connected in $G_{k}^{t} G_{k}-I$. But the entry $\left(b_{2} b_{1}, a_{2} a_{1}\right)$ does not correspond to a cell, since $b_{2}$ and $a_{2}$ are not connected in $G_{k} G_{k}^{t}-I$. To simplify the computations of the absolute values of the entries of $U$ we label the entries corresponding to cells. We choose to label the three $3 \times 3$ blocks in Table 1 different from the rest, because they are special in that only elements from these blocks are part of a $3 \times 3$ unitary block of either $U$ or $V$. In the upper left $3 \times 3$ 
block every element corresponds to two cells due to the double $d d$ edge. We label the cells with the edge $d \stackrel{\alpha}{-} d$ by $f$, and the cells with the edge $d \stackrel{\gamma}{-} d$ by $g$. The rest of the cells are labeled as if Table 1 was a matrix denoted by $Z$.

The aim is to show that we can find unitary matrices $U$ and $V$ satisfying the bi-unitary condition in Theorem 2.3 such that $U$ is symmetric under $\phi$. This means that we must have

$$
u_{(j, \rho, \sigma),(l, \zeta, \psi)}^{(i, k)}=u_{(\phi(j), \phi(\rho), \phi(\sigma)),(\phi(l), \phi(\sigma), \phi(\psi))}^{(\phi(i), \phi(k))}
$$

for all $(i, j, k, \rho, \sigma) \in S$ and all $(i, l, k, \zeta, \psi) \in T$. So for instance the entries of $U$ labeled by $\left(b_{2} b_{1}, d b_{1}\right)$ and by $\left(a_{2} a_{1}, d a_{1}\right)$ are the same. In the upper left $3 \times 3$ corner we have the double $d d$ edge, hence we have a choice of whether to define the automorphism $\phi$ such that it switches the edges or such that it leaves them invariant. It turns out that we have to let $\phi$ switch these edges, because otherwise we end up with a block of $V$ with two equal columns when we use the symmetry, hence a symmetric connection under such a $\phi$ cannot be found. Thus $\phi$ maps the edge $d \stackrel{\alpha}{-} d$ to the edge $d \stackrel{\gamma}{-} d$ and vice versa. Taking this into account we get the labeling of the entries of $U$ in Table 1 .

\begin{tabular}{|c||c|c|c||c|c|c||c|c|c|c|c|}
\hline \multicolumn{10}{|c|}{ Table 1: Labels of the entries of the connection $U$} \\
\hline Cells & $d a_{1}$ & $d b_{1}$ & $d c_{1}$ & $a_{2} a_{1}$ & $b_{2} b_{1}$ & $c_{2} c_{1}$ & $a_{2} a_{3}$ & $b_{2} b_{3}$ & $c_{2} c_{3}$ & $a_{4} a_{3}$ & $b_{4} b_{3}$ \\
\hline$d a_{1}$ & $f_{11} g_{11}$ & $f_{12} g_{12}$ & $f_{13} g_{13}$ & $\sigma_{11}$ & $\sigma_{12}$ & $\sigma_{13}$ & $z_{17}$ & & & & \\
\hline$d b_{1}$ & $g_{12} f_{12}$ & $g_{11} f_{11}$ & $g_{13} f_{13}$ & $\sigma_{12}$ & $\sigma_{11}$ & $\sigma_{13}$ & & $z_{17}$ & & & \\
\hline$d c_{1}$ & $f_{31} g_{31}$ & $g_{31} f_{31}$ & $f_{33} f_{33}$ & $\sigma_{31}$ & $\sigma_{31}$ & $\sigma_{33}$ & & & $z_{39}$ & & \\
\hline \hline$a_{2} a_{1}$ & $\tau_{11}$ & $\tau_{12}$ & $\tau_{13}$ & $z_{44}$ & & & $z_{47}$ & & & $z_{4,10}$ & \\
\hline$b_{2} b_{1}$ & $\tau_{12}$ & $\tau_{11}$ & $\tau_{13}$ & & $z_{44}$ & & & $z_{47}$ & & & $z_{4,10}$ \\
\hline$c_{2} c_{1}$ & $\tau_{31}$ & $\tau_{31}$ & $\tau_{33}$ & & & $z_{66}$ & & & $z_{69}$ & & \\
\hline \hline$a_{2} a_{3}$ & $z_{71}$ & & & $z_{74}$ & & & $z_{77}$ & & & $z_{7,10}$ & \\
\hline$b_{2} b_{3}$ & & $z_{71}$ & & & $z_{74}$ & & & $z_{77}$ & & & $z_{7,10}$ \\
\hline$c_{2} c_{3}$ & & & $z_{93}$ & & & $z_{96}$ & & & & & \\
\hline$a_{4} a_{3}$ & & & & $z_{10,4}$ & & & $z_{10,7}$ & & & & \\
\hline$b_{4} b_{3}$ & & & & & $z_{10,4}$ & & & $z_{10,7}$ & & & \\
\hline
\end{tabular}

This table illustrates the example when $k=4$, i.e. corresponding to the graph $\Gamma_{4}$. It can easily be generalized since we note that the three upper left $3 \times 3$ blocks are special, but the rest of the table follows a pattern. It gives us five "diagonal" rays, the "real" diagonal, which we will refer to as the diagonal, the two "inner" ones, which we will refer to as the inner diagonals, and the two "outer" ones, which we will refer to as the outer diagonals. (For general $k$ we have a total of $2 k+(k-1)=3 k-1$ edges of $\Gamma_{k}$, so the table is $(3 k-1) \times(3 k-1)$.) Let $a_{0}=b_{0}=c_{0}=d$. Then all the cells outside the upper left $6 \times 6$ block have labels of the form $\left(x_{i} x_{j}, x_{k} x_{l}\right)$, where $x_{i}$ is either all $a_{i}$, all $b_{i}$, or all $c_{i}$, and $i, j, k, l$ are indices, $|i-j|=1,|k-l|=1$ (this just means that we have an edge) and $|i-k| \in\{0,2\},|j-l| \in\{0,2\}$. Note that the last three elements on the diagonal do not correspond to cells, since $\left(G_{k} G_{k}^{t}-I\right)_{p p}=0$ when $p$ is an endpoint of $\Gamma_{k}$. This gives a labeling of the cells for general $k$.

The block structures of the matrices $U$ and $V$ are given in Tables 2 and 3, where entries labeled with the same letter denote entries of the same block matrix of $U$, respectively $V$, and cells labeled with $\bullet$ are $1 \times 1$ blocks. In order to find the block $u^{(i, k)}$ of $U$, we fix the left vertex in the row at $i$ and the right vertex in the column at $k$, and vary the rest within the table. For $v^{(j, l)}$ we fix the right vertex in the row at $j$ and the left vertex in the column at $l$ and vary the rest. 


\begin{tabular}{|c|c|c|c|c|c|c|c|c|c|c|c|}
\hline \multicolumn{12}{|c|}{ Table 2: Block structure of $U$} \\
\hline Cells & $d a_{1}$ & $d b_{1}$ & $d c_{1}$ & $a_{2} a_{1}$ & $b_{2} b_{1}$ & $c_{2} c_{1}$ & $a_{2} a_{3}$ & $b_{2} b_{3}$ & $c_{2} c_{3}$ & $a_{4} a_{3}$ & $b_{4} b_{3}$ \\
\hline$d a_{1}$ & $\bar{a} a$ & $b b$ & $c c$ & $a$ & $b$ & $c$ & $\bullet$ & & & & \\
\hline$d b_{1}$ & $a a$ & $b b$ & $c c$ & $a$ & $b$ & $c$ & & - & & & \\
\hline$\frac{d c_{1}}{}$ & $a a$ & $b b$ & $\overline{c c}$ & $a$ & $\bar{b}$ & $c$ & & & - & & \\
\hline$a_{2} a_{1}$ & $d$ & - & - & $d$ & & & $g$ & & & $g$ & \\
\hline$b_{2} b_{1}$ & - & $\bar{e}$ & - & & $e$ & & & $h$ & & & $h$ \\
\hline$c_{2} c_{1}$ & - & - & $f$ & & & $f$ & & & - & & \\
\hline$a_{2} a_{3}$ & $d$ & & & $d$ & & & $g$ & & & $g$ & \\
\hline$b_{2} b_{3}$ & & $e$ & & & $e$ & & & $h$ & & & $h$ \\
\hline$c_{2} c_{3}$ & & & $f$ & & & $f$ & & & & & \\
\hline$a_{4} a_{3}$ & & & & - & & & - & & & & \\
\hline$b_{4} b_{3}$ & & & & & - & & & - & & & \\
\hline
\end{tabular}

\begin{tabular}{|c|c|c|c|c|c|c|c|c|c|c|c|}
\hline \multicolumn{12}{|c|}{ Table 3: Block structure of $V$} \\
\hline Cells & $d a_{1}$ & $d b_{1}$ & $d c_{1}$ & $a_{2} a_{1}$ & $b_{2} b_{1}$ & $\overline{c_{2} c_{1}}$ & $a_{2} a_{3}$ & $b_{2} b_{3}$ & $c_{2} c_{3}$ & $a_{4} a_{3}$ & $b_{4} b_{3}$ \\
\hline$d a_{1}$ & $a a^{2}$ & $\overline{a d a}$ & $a d$ & $d$ & $\bullet$ & $\bullet$ & $d$ & & & & \\
\hline$d b_{1}$ & $\overline{b b}$ & $\overline{b b}$ & $b b$ & $\bullet$ & $\bar{e}$ & $\bullet$ & & $e$ & & & \\
\hline$d c_{1}$ & $c c$ & $c c$ & $c c$ & $\bullet$ & $\bullet$ & $f$ & & & $f$ & & \\
\hline$a_{2} a_{1}$ & $a$ & $a$ & $a$ & $\bar{d}$ & & & $d$ & & & $\bullet$ & \\
\hline$b_{2} b_{1}$ & $b$ & $b$ & $b$ & & $e$ & & & $e$ & & & $\bullet$ \\
\hline$c_{2} c_{1}$ & $\bar{c}$ & $\bar{c}$ & $\bar{c}$ & & & $f$ & & & $f$ & & \\
\hline$a_{2} a_{3}$ & $\bullet$ & & & $g$ & & & $g$ & & & $\bullet$ & \\
\hline$b_{2} b_{3}$ & & $\bullet$ & & & $h$ & & & $h$ & & & $\bullet$ \\
\hline$c_{2} c_{3}$ & & & - & & & $\bullet$ & & & & & \\
\hline$a_{4} a_{3}$ & & & & $g$ & & & $g$ & & & & \\
\hline$b_{4} b_{3}$ & & & & & $h$ & & & $h$ & & & \\
\hline
\end{tabular}

For instance we get that the block labeled as

$$
\left(\begin{array}{cc}
\left(a_{2} a_{1}, a_{2} a_{3}\right) & \left(a_{2} a_{1}, a_{4} a_{3}\right) \\
\left(a_{2} a_{3}, a_{2} a_{3}\right) & \left(a_{2} a_{3}, a_{4} a_{3}\right)
\end{array}\right)=\left(\begin{array}{cc}
z_{47} & z_{4,10} \\
z_{77} & z_{7,10}
\end{array}\right)
$$

is an example of a $2 \times 2$ block of $U$.

Finally we for instance get the coefficient labeled by the cell $\left(d a_{1}, a_{2} a_{1}\right)$ as

$$
\sqrt{\frac{\xi(d) \xi\left(a_{1}\right)}{\xi\left(a_{1}\right) \xi\left(a_{2}\right)}}=\sqrt{\frac{1}{\xi\left(a_{2}\right)}} .
$$

Note that the coefficient of entry $(i, j)$ is the inverse of the coefficient of entry $(j, i)$, when the table is considered a matrix. This symmetry will be called the inverse diagonal symmetry of the coefficients, and will be used later on.

3.1.2. Absolute values. Now we find absolute values for all the entries of the matrix $U$. We start out by working with the $3 \times 3$ blocks, which we obtain from Tables 2 and 3 . The $3 \times 3$ blocks of $U$ are

$$
\left(\begin{array}{lll}
f_{11} & g_{11} & \sigma_{11} \\
g_{12} & f_{12} & \sigma_{12} \\
f_{31} & g_{31} & \sigma_{31}
\end{array}\right),\left(\begin{array}{lll}
f_{12} & g_{12} & \sigma_{12} \\
g_{11} & f_{11} & \sigma_{11} \\
g_{31} & f_{31} & \sigma_{31}
\end{array}\right),\left(\begin{array}{lll}
f_{13} & g_{13} & \sigma_{13} \\
g_{13} & f_{13} & \sigma_{13} \\
f_{33} & f_{33} & \sigma_{33}
\end{array}\right)
$$


and the $3 \times 3$ blocks of $V$ are

$$
\left(\begin{array}{lll}
\widetilde{f}_{11} & \widetilde{f}_{12} & \widetilde{f}_{13} \\
\widetilde{g}_{11} & \widetilde{g}_{12} & \widetilde{g}_{13} \\
\widetilde{\tau}_{11} & \widetilde{\tau}_{12} & \widetilde{\tau}_{13}
\end{array}\right),\left(\begin{array}{lll}
\widetilde{g}_{12} & \widetilde{g}_{11} & \widetilde{g}_{13} \\
\widetilde{f}_{12} & \widetilde{f}_{11} & \widetilde{f}_{13} \\
\widetilde{\tau}_{12} & \widetilde{\tau}_{11} & \widetilde{\tau}_{13}
\end{array}\right),\left(\begin{array}{lll}
\widetilde{f}_{31} & \widetilde{g}_{31} & \widetilde{f}_{33} \\
\widetilde{g}_{31} & \widetilde{f}_{31} & \widetilde{f}_{33} \\
\widetilde{\tau}_{31} & \widetilde{\tau}_{31} & \widetilde{\tau}_{33}
\end{array}\right),
$$

where we use a tilde to denote the entry of $U$ up to multiplication by the coefficient from Theorem [2.3. This coefficient we denote by $\zeta_{x, i j}$, i.e. $\tilde{x}_{i j}=\zeta_{x, i j} x_{i j}$ for $x \in$ $\{f, g, \tau, \sigma, z\}$. Note that the first two blocks of $U$, respectively $V$, are the same up to permutations of the rows and columns, hence we only have to deal with four $3 \times 3$ blocks.

From the block structure of $U$ and $V$ we get that $\left|\tau_{i j}\right|=1=\left|\tilde{\sigma}_{i j}\right|$ for $i \neq j$. We compute $\left|\tau_{11}\right|,\left|\tau_{33}\right|,\left|\sigma_{11}\right|$ and $\left|\sigma_{33}\right|$ from these $3 \times 3$ blocks by using the fact that their rows and columns are orthonormal. We get

$$
\begin{aligned}
\left|\sigma_{11}\right|^{2} & =1-\left|\sigma_{12}\right|^{2}-\left|\sigma_{31}\right|^{2}=1-\zeta_{\sigma, 12}^{-2}-\zeta_{\sigma, 31}^{-2}=1-\xi\left(a_{2}\right)-\frac{\xi\left(c_{1}\right) \xi\left(a_{2}\right)}{\xi\left(a_{1}\right)}, \\
\left|\sigma_{33}\right|^{2} & =1-2\left|\sigma_{13}\right|^{2}=1-2 \zeta_{\sigma, 13}^{-2}=1-2 \frac{\xi\left(a_{1}\right) \xi\left(c_{2}\right)}{\xi\left(c_{1}\right)} \\
\left|\tau_{11}\right|^{2} & =\zeta_{\tau, 11}^{-2}\left(1-\left|\widetilde{\tau}_{12}\right|^{2}-\left|\widetilde{\tau}_{13}\right|^{2}\right)=\zeta_{\tau, 11}^{-2}\left(1-\zeta_{\tau, 12}^{2}-\zeta_{\tau, 13}^{2}\right) \\
& =\frac{1}{\xi\left(a_{2}\right)}\left(1-\xi\left(a_{2}\right)-\frac{\xi\left(c_{1}\right) \xi\left(a_{2}\right)}{\xi\left(a_{1}\right)}\right)=\frac{1}{\xi\left(a_{2}\right)}-1-\frac{\xi\left(c_{1}\right)}{\xi\left(a_{1}\right)} \\
\left|\tau_{33}\right|^{2} & =\zeta_{\tau, 33}^{-2}\left(1-2\left|\widetilde{\tau}_{31}\right|^{2}\right)=\zeta_{\tau, 33}^{-2}\left(1-2 \zeta_{\tau, 31}^{2}\right) \\
& =\frac{1}{\xi\left(c_{2}\right)}\left(1-2 \frac{\xi\left(a_{1}\right) \xi\left(c_{2}\right)}{\xi\left(c_{1}\right)}\right)=\frac{1}{\xi\left(c_{2}\right)}-2 \frac{\xi\left(a_{1}\right)}{\xi\left(c_{1}\right)} .
\end{aligned}
$$

Note that all these values are positive so this makes sense. Positivity follows by using Lemma 3.1 and Lemma 3.2 , which we will prove later.

Let $x_{i j}^{\circ}$ denote the complex phase of the element $x_{i j}, x \in\{f, g, \tau, \sigma, z\}$. Then we have

$$
\begin{aligned}
& \tau=\left(\begin{array}{ccc}
\zeta_{\tau, 11}^{-1} \sqrt{1-\zeta_{\tau, 12}^{2}-\zeta_{\tau, 13}^{2}} \stackrel{\circ}{\tau}_{11} & \stackrel{\circ}{\tau}_{12} & \stackrel{\circ}{\tau}_{13} \\
\stackrel{\circ}{\tau}_{12} & \zeta_{\tau, 11}^{-1} \sqrt{1-\zeta_{\tau, 12}^{2}-\zeta_{\tau, 13}^{2}} \stackrel{\circ}{\tau}_{11} & \stackrel{\circ}{\tau}_{13} \\
\stackrel{\circ}{\tau}_{31} & \stackrel{\circ}{\tau}_{31} & \zeta_{\tau, 33}^{-1} \sqrt{1-2 \zeta_{\tau, 31}^{2}} \circ_{\tau_{33}}
\end{array}\right),
\end{aligned}
$$

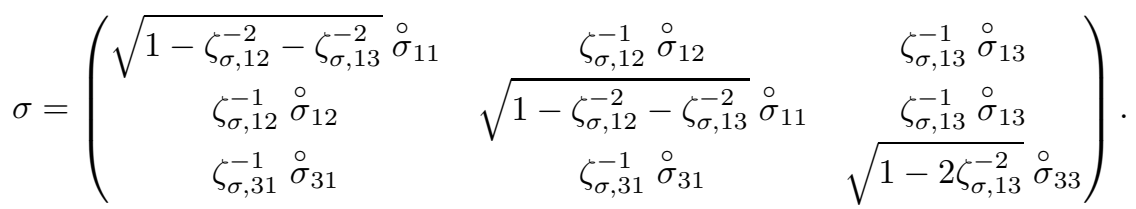

The inverse diagonal symmetry of the coefficients gives $\zeta_{\tau, i j}=\zeta_{\sigma, j i}^{-1}$. Using this we get

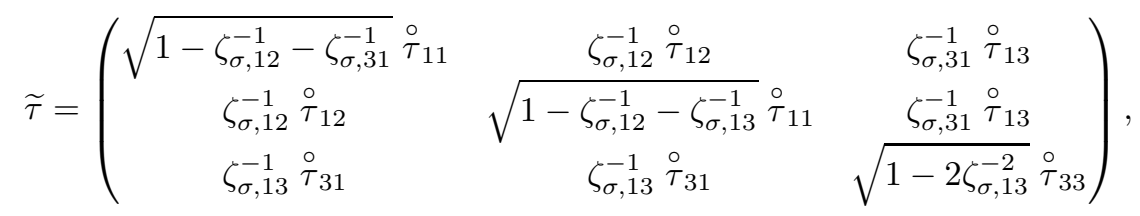


thus $\left|\widetilde{\tau}_{12}\right|=\left|\sigma_{12}\right|,\left|\widetilde{\tau}_{13}\right|=\left|\sigma_{31}\right|$, and $\left|\widetilde{\tau}_{31}\right|=\left|\sigma_{13}\right|$. We see that this implies

$$
\left|\widetilde{\sigma}_{i i}\right|=\left|\tau_{i i}\right|
$$

for $i=1,3$. We will see later that the same symmetry holds for the remaining entries of $U$. Now let us work with the $3 \times 3$ blocks $A, B, C$, and $D$ given as

$$
\begin{aligned}
& A=\left(\begin{array}{lll}
f_{11} & g_{11} & \sigma_{11} \\
g_{12} & f_{12} & \sigma_{12} \\
f_{31} & g_{31} & \sigma_{31}
\end{array}\right), \quad B=\left(\begin{array}{lll}
f_{13} & g_{13} & \sigma_{13} \\
g_{13} & f_{13} & \sigma_{13} \\
f_{33} & f_{33} & \sigma_{33}
\end{array}\right), \\
& C=\left(\begin{array}{ccc}
\widetilde{f}_{11} & \widetilde{f}_{12} & \widetilde{f}_{13} \\
\widetilde{g}_{11} & \widetilde{g}_{12} & \widetilde{g}_{13} \\
\widetilde{\tau}_{11} & \widetilde{\tau}_{12} & \widetilde{\tau}_{13}
\end{array}\right)=\left(\begin{array}{ccc}
f_{11} & f_{12} & \zeta_{f, 13} f_{13} \\
g_{11} & g_{12} & \zeta_{f, 13} g_{13} \\
\left|\sigma_{11}\right| \stackrel{\circ}{1}_{11} & \left|\sigma_{12}\right| \stackrel{\circ}{12}_{12} & \left|\sigma_{31}\right| \stackrel{\circ}{13}_{13}
\end{array}\right), \\
& D=\left(\begin{array}{ccc}
\widetilde{f}_{31} & \widetilde{g}_{31} & \widetilde{f}_{33} \\
\widetilde{g}_{31} & \widetilde{f}_{31} & \widetilde{f}_{33} \\
\widetilde{\tau}_{31} & \widetilde{\tau}_{31} & \widetilde{\tau}_{33}
\end{array}\right)=\left(\begin{array}{ccc}
\zeta_{f, 31} f_{31} & \zeta_{f, 31} g_{31} & f_{33} \\
\zeta_{f, 31} g_{31} & \zeta_{f, 31} f_{31} & f_{33} \\
\left|\sigma_{13}\right| \stackrel{\tau}{31}_{31} & \left|\sigma_{13}\right| \dot{\tau}_{31} & \left|\sigma_{33}\right| \stackrel{\circ}{\tau}_{33}
\end{array}\right) .
\end{aligned}
$$

Note the similarities between $A$ and $C$, and between $B$ and $D$. First consider $B$. We have

$$
\left|f_{33}\right|^{2}=\frac{1}{2}\left(1-\left|\sigma_{33}\right|^{2}\right)=\frac{1}{2}\left(1-\left(1-2 \frac{\xi\left(a_{1}\right) \xi\left(c_{2}\right)}{\xi\left(c_{1}\right)}\right)\right)=\frac{\xi\left(a_{1}\right) \xi\left(c_{2}\right)}{\xi\left(c_{1}\right)}=\left|\sigma_{13}\right|^{2} .
$$

So if we let $\left|f_{13}\right|=\left|\sigma_{33}\right|$, and $\left|g_{13}\right|=\left|\sigma_{13}\right|$, then the rows and columns of $B$ are normalized. Similarly $D$ has normalized rows and columns if we let

$$
\begin{aligned}
& \left|f_{31}\right|^{2}=\zeta_{f, 31}^{-2}\left|\sigma_{33}\right|^{2}=\frac{\xi\left(c_{1}\right)}{\xi\left(a_{1}\right)}\left(1-2 \frac{\xi\left(a_{1}\right) \xi\left(c_{2}\right)}{\xi\left(c_{1}\right)}\right), \\
& \left|g_{31}\right|^{2}=\zeta_{f, 31}^{-2}\left|\sigma_{13}\right|^{2}=\frac{\xi\left(c_{1}\right)}{\xi\left(a_{1}\right)}\left(\frac{\xi\left(a_{1}\right) \xi\left(c_{2}\right)}{\xi\left(c_{1}\right)}\right)=\xi\left(c_{2}\right) .
\end{aligned}
$$

For $A$ and $C$ these choices give

$$
\begin{aligned}
& A=\left(\begin{array}{ccc}
f_{11} & g_{11} & \sqrt{\left(1-\xi\left(a_{2}\right)-\frac{\xi\left(c_{1}\right) \xi\left(a_{2}\right)}{\xi\left(a_{1}\right)}\right)} \sigma_{11} \\
g_{12} & f_{12} & \sqrt{\xi\left(a_{2}\right) \sigma_{12}^{\circ}} \\
\sqrt{\frac{\xi\left(c_{1}\right)}{\xi\left(a_{1}\right)}\left(1-2 \frac{\xi\left(a_{1}\right) \xi\left(c_{2}\right)}{\xi\left(c_{1}\right)}\right)} f_{31} & \sqrt{\xi\left(c_{2}\right)} g_{31}^{\circ} & \sqrt{\frac{\xi\left(c_{1}\right) \xi\left(a_{2}\right)}{\xi\left(a_{1}\right)} \sigma_{31}}
\end{array}\right), \\
& C=\left(\begin{array}{ccc}
f_{11} & f_{12} & \sqrt{\frac{\xi\left(c_{1}\right)}{\xi\left(a_{1}\right)}\left(1-2 \frac{\xi\left(a_{1}\right) \xi\left(c_{2}\right)}{\xi\left(c_{1}\right)}\right)} f_{13} \\
g_{11} & g_{12} & \sqrt{\xi\left(c_{2}\right)} \stackrel{\circ}{13}^{\circ} \\
\sqrt{\left(1-\xi\left(a_{2}\right)-\frac{\xi\left(c_{1}\right) \xi\left(a_{2}\right)}{\xi\left(a_{1}\right)}\right)} \stackrel{\circ}{\tau}_{11} & \sqrt{\xi\left(a_{2}\right) \stackrel{\circ}{\tau}_{12}} & \sqrt{\frac{\xi\left(c_{1}\right) \xi\left(a_{2}\right)}{\xi\left(a_{1}\right)} \stackrel{\circ}{\tau}_{13}}
\end{array}\right) .
\end{aligned}
$$

So for both $A$ and $C$ to be unitary, we must have $\left|f_{12}\right|=\left|g_{12}\right|$, hence $\left|f_{12}\right|^{2}=$ $\left|g_{12}\right|^{2}=\frac{1}{2}\left(1-\xi\left(a_{2}\right)\right)$. This implies that

$$
\begin{aligned}
& \left|f_{11}\right|^{2}=1-\frac{1}{2}\left(1-\xi\left(a_{2}\right)\right)-\frac{\xi\left(c_{1}\right)}{\xi\left(a_{1}\right)}+2 \xi\left(c_{2}\right), \\
& \left|g_{11}\right|^{2}=1-\left|f_{12}\right|^{2}-\left|g_{31}\right|^{2}=1-\frac{1}{2}\left(1-\xi\left(a_{2}\right)\right)-\xi\left(c_{2}\right) .
\end{aligned}
$$

Note again that all these values are positive by Lemma 3.1 and Lemma 3.2. In order for $A$ and $C$ to be unitary we need to check that $\left|f_{11}\right|^{2}+\left|g_{11}\right|^{2}+\left|\sigma_{11}\right|^{2}=1$. This is done using equations (3.4) and (3.5). 
This shows that the rows and columns of $A$ and $C$ are normalized. Now we have determined the absolute values of the entries in the three upper left $3 \times 3$ blocks of the table, and we are only left with determining the absolute values of the entries $z_{i j}$. They either belong to some $2 \times 2$ block of $U$ or of $V$. For given $k$ we have, by letting $a_{0}=b_{0}=c_{0}=d$, that all the $2 \times 2$ blocks of $U$ are of the form

$$
\left(\begin{array}{cc}
\left(x_{i} x_{i-1}, x_{i-2} x_{i-1}\right) & \left(x_{i} x_{i-1}, x_{i} x_{i-1}\right) \\
\left(x_{i} x_{i+1}, x_{i-2} x_{i-1}\right) & \left(x_{i} x_{i+1}, x_{i} x_{i-1}\right)
\end{array}\right),\left(\begin{array}{cc}
\left(x_{i} x_{i-1}, x_{i} x_{i+1}\right) & \left(x_{i} x_{i-1}, x_{i+2} x_{i+1}\right) \\
\left(x_{i} x_{i+1}, x_{i} x_{i+1}\right) & \left(x_{i} x_{i+1}, x_{i+2} x_{i+1}\right)
\end{array}\right),
$$

where either $x_{i}=a_{i}$ for all $i$, or $x_{i}=b_{i}$ for all $i$, or $x_{i}=c_{i}$ for all $i$, and $i$ is even.

This means that for each even $i, 1 \leq i \leq k$, we have a $2 \times 2$ block of $U$ whenever the vertex $x_{i+1}$ of $\Gamma_{k}$ exists, and another $2 \times 2$ block whenever the vertex $x_{i+2}$ also exists. This gives a total of $(k-2) 2 \times 2$ blocks for both $x=a$ and $x=b$. For $x=c$ we get $(k-3) 2 \times 2$ blocks, since the $c$ ray has length $k-1$. So a priori we have a total of $2(k-2)+(k-3) 2 \times 2$ blocks of $U$. But since we have that the connection is symmetric under $\phi$, the $2 \times 2$ blocks arising as above labeled by the $a$-ray, respectively the $b$-ray, are the same; see Table 1 . For instance we have

$$
\left(\begin{array}{ll}
\left(a_{2} a_{1}, a_{2} a_{3}\right) & \left(a_{2} a_{1}, a_{4} a_{3}\right) \\
\left(a_{2} a_{3}, a_{2} a_{3}\right) & \left(a_{2} a_{3}, a_{4} a_{3}\right)
\end{array}\right)=\left(\begin{array}{ll}
z_{47} & z_{4,10} \\
z_{77} & z_{7,10}
\end{array}\right)=\left(\begin{array}{ll}
\left(b_{2} b_{1}, b_{2} b_{3}\right) & \left(b_{2} b_{1}, b_{4} b_{3}\right) \\
\left(b_{2} b_{3}, b_{2} b_{3}\right) & \left(b_{2} b_{3}, b_{4} b_{3}\right)
\end{array}\right) .
$$

Thus we are left with a total of $(k-2)+(k-3) 2 \times 2$ blocks of $U$. The total number of $2 \times 2$ blocks of $V$ is the same. In the case of $k=4$ we get the following $2 \times 2$ blocks of $U$ :

$$
\left(\begin{array}{ll}
\tau_{11} & z_{44} \\
z_{71} & z_{74}
\end{array}\right),\left(\begin{array}{ll}
\tau_{33} & z_{66} \\
z_{93} & z_{96}
\end{array}\right),\left(\begin{array}{ll}
z_{47} & z_{4,10} \\
z_{77} & z_{7,10}
\end{array}\right)
$$

and the following $2 \times 2$ blocks of $V$ :

$$
\left(\begin{array}{cc}
\left|\tau_{11}\right| \stackrel{\circ}{\sigma 1}_{11} & \widetilde{z}_{17} \\
z_{44} & \widetilde{z}_{47}
\end{array}\right),\left(\begin{array}{cc}
\left|\tau_{33}\right| \stackrel{\circ}{\sigma}_{33} & \widetilde{z}_{39} \\
z_{66} & \widetilde{z}_{69}
\end{array}\right),\left(\begin{array}{cc}
\widetilde{z}_{74} & z_{77} \\
\widetilde{z}_{10,4} & \widetilde{z}_{10,7}
\end{array}\right)
$$

by using equation (3.7) and the coefficients. Let

$$
A=\left(\begin{array}{cc}
\tau_{11} & z_{44} \\
z_{71} & z_{74}
\end{array}\right), \quad B=\left(\begin{array}{cc}
\left|\tau_{11}\right| \stackrel{\circ}{\sigma}_{11} & \widetilde{z}_{17} \\
z_{44} & \widetilde{z}_{47}
\end{array}\right)
$$

For both of these matrices to be unitary we must have that $\left|z_{71}\right|=\left|\widetilde{z}_{17}\right|$ and $\left|z_{74}\right|=\left|\widetilde{z}_{47}\right|$. This is part of the general pattern we get by comparing the $2 \times 2$ blocks of $U$ to the $2 \times 2$ blocks of $V$, which gives

$$
\left|z_{i j}\right|=\left|\widetilde{z}_{j i}\right|
$$

for all $i$ and $j$, by remembering that the coefficients have inverse symmetry across the diagonal. So by using this it is enough to compute the absolute values of the entries of the $2 \times 2$ blocks of $U$. From the first one we get

$$
\left|z_{44}\right|^{2}=\left|z_{71}\right|^{2}=1-\left|\tau_{11}\right|^{2}, \quad\left|z_{74}\right|=\left|\tau_{11}\right|,
$$

and from for the second one we get

$$
\left|z_{93}\right|^{2}=\left|z_{66}\right|^{2}=1-\left|\tau_{33}\right|^{2}, \quad\left|z_{96}\right|=\left|\tau_{33}\right| .
$$

We can continue this way, since in each $2 \times 2$ block we have already determined the absolute value of the entry in the upper left corner, which then determines the absolute values of the last three entries, since the rows and the columns have to be normalized. By using the fact that $\left|z_{i j}\right|=\left|\widetilde{z}_{j i}\right|$ and the coefficients, we find all the absolute values of the entries of $U$. 
3.1.3. Consistency of the assigned absolute values. We have found the absolute values of the entries of $U$, such that all $3 \times 3$ and $2 \times 2$ blocks of $U$ and of $V$ have rows and columns normalized. We need to also check that the $1 \times 1$ blocks of $U$ and of $V$ have absolute value one. The entries of these $1 \times 1$ blocks appear in Table 1 on the outer diagonals and as the last two entries on the inner diagonals at the lower right corner corresponding to the end points of the rays (see Tables 2 and 3 ). Let us work our way down to find the pattern. By using alternating $2 \times 2$ blocks of $U$ and of $V$, we see that

$$
\left|\tau_{11}\right|=\left|z_{74}\right|, \quad\left|\widetilde{z}_{74}\right|=\left|\widetilde{z}_{10,7}\right|, \quad\left|z_{10,7}\right|=\left|z_{13,10}\right|, \quad\left|\widetilde{z}_{13,10}\right|=\left|\widetilde{z}_{16,13}\right| \ldots
$$

and

$$
\left|\tau_{33}\right|=\left|z_{96}\right|, \quad\left|\widetilde{z}_{96}\right|=\left|\widetilde{z}_{12,9}\right|, \quad\left|z_{12,9}\right|=\left|z_{15,12}\right|, \quad\left|\widetilde{z}_{15,12}\right|=\left|\widetilde{z}_{18,15}\right| \ldots,
$$

hence by using the coefficients we get

$$
\begin{aligned}
\frac{1}{\xi\left(a_{2}\right)}-1-\frac{\xi\left(c_{1}\right)}{\xi\left(a_{1}\right)} & =\left|\tau_{11}\right|^{2}=\left|z_{74}\right|^{2}=\frac{\xi\left(a_{3}\right)}{\xi\left(a_{1}\right)}\left|\widetilde{z}_{74}\right|^{2}=\frac{\xi\left(a_{3}\right)}{\xi\left(a_{1}\right)}\left|\widetilde{z}_{10,7}\right|^{2} \\
& =\frac{\xi\left(a_{3}\right) \xi\left(a_{4}\right)}{\xi\left(a_{1}\right) \xi\left(a_{2}\right)}\left|z_{10,7}\right|^{2}=\cdots=\frac{\xi\left(a_{3}\right) \xi\left(a_{4}\right)}{\xi\left(a_{1}\right) \xi\left(a_{2}\right)}\left|z_{13,10}\right|^{2} \\
& =\frac{\xi\left(a_{3}\right) \xi\left(a_{4}\right) \xi\left(a_{5}\right)}{\xi\left(a_{1}\right) \xi\left(a_{2}\right) \xi\left(a_{3}\right)}\left|\widetilde{z}_{13,10}\right|^{2}=\frac{\xi\left(a_{4}\right) \xi\left(a_{5}\right)}{\xi\left(a_{1}\right) \xi\left(a_{2}\right)}\left|\widetilde{z}_{16,13}\right|^{2} \\
& =\frac{\xi\left(a_{4}\right) \xi\left(a_{5}\right) \xi\left(a_{6}\right)}{\xi\left(a_{1}\right) \xi\left(a_{2}\right) \xi\left(a_{4}\right)}\left|z_{16,10}\right|^{2}=\frac{\xi\left(a_{5}\right) \xi\left(a_{6}\right)}{\xi\left(a_{1}\right) \xi\left(a_{2}\right)}\left|z_{19,16}\right|^{2} \ldots
\end{aligned}
$$

and

$$
\begin{aligned}
\frac{1}{\xi\left(c_{2}\right)}-2 \frac{\xi\left(a_{1}\right)}{\xi\left(c_{1}\right)} & =\left|\tau_{33}\right|^{2}=\left|z_{96}\right|^{2}=\frac{\xi\left(c_{3}\right)}{\xi\left(c_{1}\right)}\left|\widetilde{z}_{96}\right|^{2} \\
& =\frac{\xi\left(c_{3}\right)}{\xi\left(c_{1}\right)}\left|\widetilde{z}_{12,9}\right|^{2}=\frac{\xi\left(c_{3}\right) \xi\left(c_{4}\right)}{\xi\left(c_{1}\right) \xi\left(c_{2}\right)}\left|z_{15,12}\right|^{2}=\ldots \\
& =\frac{\xi\left(c_{3}\right) \xi\left(c_{4}\right) \xi\left(c_{5}\right)}{\xi\left(c_{1}\right) \xi\left(c_{2}\right) \xi\left(c_{3}\right)}\left|\widetilde{z}_{18,15}\right|^{2}=\frac{\xi\left(c_{4}\right) \xi\left(c_{5}\right)}{\xi\left(c_{1}\right) \xi\left(c_{2}\right)}\left|\widetilde{z}_{18,15}\right|^{2} \ldots
\end{aligned}
$$

Given $k$ we have that Table 1 is $(3 k-1) \times(3 k-1)$, hence the entries on the lower inner diagonal, which are $1 \times 1$ blocks in $U$, are $z_{(3 k-2),(3 k-5)}$ and $z_{(3 k-3),(3 k-6)}$ corresponding to the cells $\left(a_{k} a_{k-1}, a_{k-2} a_{k-1}\right)$ and $\left(c_{k-2} c_{k-1}, c_{k-2} c_{k-3}\right)$. In fact we have four such elements, two on each inner diagonal, where the two ones on the upper inner diagonal are $1 \times 1$ blocks in $V$. But again due to symmetry, it is enough to work with the two on the lower inner diagonal. By relating them to either $\tau_{11}$ or $\tau_{33}$ as above, we arrive at the following two equations, which must be satisfied in order for our construction to give a symmetric connection:

$$
\begin{aligned}
\xi\left(a_{1}\right)-\xi\left(a_{1}\right) \xi\left(a_{2}\right)-\xi\left(c_{1}\right) \xi\left(a_{2}\right) & =\xi\left(a_{k-1}\right) \xi\left(a_{k}\right), \\
\xi\left(c_{1}\right)-2 \xi\left(a_{1}\right) \xi\left(c_{2}\right) & =\xi\left(c_{k-2}\right) \xi\left(c_{k-1}\right) .
\end{aligned}
$$

We will show later that these identities are satisfied.

Let us find the other identities which must be satisfied. Consider the outer diagonal elements. We know that they are $1 \times 1$ blocks in either $U$ or $V$, but we have also found their absolute values in terms of the absolute value of an inner diagonal element by using that they are part of a $2 \times 2$ block of either $V$ or $U$. 
Moreover we have related the absolute value of the inner diagonal elements to $\tau_{11}$ and $\tau_{33}$, respectively. Let us work through the first few cases:

1) $\left|z_{71}\right|^{2}=1-\left|\tau_{11}\right|^{2}$ and $\left|\widetilde{z}_{71}\right|=1$, so $\frac{\xi\left(a_{3}\right)}{\xi\left(a_{1}\right) \xi\left(a_{2}\right)}=1-\left(\frac{1}{\xi\left(a_{2}\right)}-1-\frac{\xi\left(c_{1}\right)}{\xi\left(a_{1}\right)}\right)$, thus

$$
\xi\left(a_{3}\right)=\xi\left(a_{1}\right) \xi\left(a_{2}\right)-\xi\left(a_{1}\right)+\xi\left(a_{1}\right) \xi\left(a_{2}\right)+\xi\left(c_{1}\right) \xi\left(a_{2}\right) .
$$

2) $\left|z_{93}\right|^{2}=1-\left|\tau_{33}\right|^{2}$ and $\left|\widetilde{z}_{93}\right|=1$, i.e. $\frac{\xi\left(c_{3}\right)}{\xi\left(c_{1}\right) \xi\left(c_{2}\right)}=1-\left(\frac{1}{\xi\left(c_{2}\right)}-2 \frac{\xi\left(a_{1}\right)}{\xi\left(c_{1}\right)}\right)$, hence

$$
\xi\left(c_{3}\right)=\xi\left(c_{1}\right) \xi\left(c_{2}\right)-\xi\left(c_{1}\right)+2 \xi\left(a_{1}\right) \xi\left(c_{2}\right) .
$$

3) $\left|\widetilde{z}_{10,4}\right|^{2}=1-\left|\widetilde{z}_{74}\right|^{2}, \quad\left|z_{10,4}\right|=1$, and $\left|z_{74}\right|=\left|\tau_{11}\right|$, i.e.

$$
\begin{aligned}
\frac{\xi\left(a_{4}\right) \xi\left(a_{1}\right)}{\xi\left(a_{2}\right) \xi\left(a_{3}\right)} & =1-\left|\widetilde{z}_{74}\right|^{2}=1-\frac{\xi\left(a_{1}\right)}{\xi\left(a_{3}\right)}\left|z_{74}\right|^{2}=1-\frac{\xi\left(a_{1}\right)}{\xi\left(a_{3}\right)}\left|\tau_{11}\right|^{2} \\
& =1-\frac{\xi\left(a_{1}\right)}{\xi\left(a_{3}\right)}\left(\frac{1}{\xi\left(a_{2}\right)}-1-\frac{\xi\left(c_{1}\right)}{\xi\left(a_{1}\right)}\right) \\
& =1-\frac{\xi\left(a_{1}\right)}{\xi\left(a_{2}\right) \xi\left(a_{3}\right)}+\frac{\xi\left(a_{1}\right)}{\xi\left(a_{3}\right)}+\frac{\xi\left(c_{1}\right)}{\xi\left(a_{3}\right)}
\end{aligned}
$$

thus

$$
\xi\left(a_{4}\right) \xi\left(a_{1}\right)=\xi\left(a_{2}\right) \xi\left(a_{3}\right)-\xi\left(a_{1}\right)+\xi\left(a_{1}\right) \xi\left(a_{2}\right)+\xi\left(c_{1}\right) \xi\left(a_{2}\right) .
$$

4) $\left|\widetilde{z}_{12,6}\right|^{2}=1-\left|\widetilde{z}_{96}\right|^{2}, \quad\left|z_{12,6}\right|=1$ and $\left|z_{96}\right|=\left|\tau_{33}\right|$, hence

$$
\begin{aligned}
\frac{\xi\left(c_{4}\right) \xi\left(c_{1}\right)}{\xi\left(c_{2}\right) \xi\left(c_{3}\right)} & =1-\left|\widetilde{z}_{96}\right|^{2}=1-\frac{\xi\left(c_{1}\right)}{\xi\left(c_{3}\right)}\left|z_{96}\right|^{2}=1-\frac{\xi\left(c_{1}\right)}{\xi\left(c_{3}\right)}\left|\tau_{33}\right|^{2} \\
& =1-\frac{\xi\left(c_{1}\right)}{\xi\left(c_{3}\right)}\left(\frac{1}{\xi\left(c_{2}\right)}-2 \frac{\xi\left(a_{1}\right)}{\xi\left(c_{1}\right)}\right)=1-\frac{\xi\left(c_{1}\right)}{\xi\left(c_{2}\right) \xi\left(c_{3}\right)}+2 \frac{\xi\left(a_{1}\right)}{\xi\left(c_{3}\right)},
\end{aligned}
$$

thus

$$
\xi\left(c_{4}\right) \xi\left(c_{1}\right)=\xi\left(c_{2}\right) \xi\left(c_{3}\right)-\xi\left(c_{1}\right)+2 \xi\left(a_{1}\right) \xi\left(c_{2}\right) .
$$

It follows that in general when the $a$-ray has length $k$ and the $c$-ray has length $k-1$, and if we let $\xi\left(a_{0}\right)=\xi\left(c_{0}\right)=1$, then we get the identities

$$
\xi\left(a_{i}\right) \xi\left(a_{i+1}\right)-\xi\left(a_{i+2}\right) \xi\left(a_{i-1}\right)=\xi\left(a_{1}\right)-\xi\left(a_{1}\right) \xi\left(a_{2}\right)-\xi\left(c_{1}\right) \xi\left(a_{2}\right)
$$

for $1 \leq i \leq k-2$ and

$$
\xi\left(c_{i}\right) \xi\left(c_{i+1}\right)-\xi\left(c_{i+2}\right) \xi\left(c_{i-1}\right)=\xi\left(c_{1}\right)-2 \xi\left(a_{1}\right) \xi\left(c_{2}\right)
$$

for $1 \leq i \leq k-3$.

Here we note that only the left-hand side depends on $i$, and that the right-hand side is the same as the left-hand side of (3.8) and (3.9). If the four identities (3.8), (3.9), (3.10) and (3.11) are satisfied, we have found consistent absolute values of the entries of the connection. In 18 Schou proved identities of the same type in order to find a connection. But since we want a connection, which is symmetric with respect to the map $\phi$, the identities we have to prove are more special. Using the facts that the $a$-ray has length $k$ and the $c$-ray has length $k-1$, and denoting $\lambda_{k}$ by $\lambda$, we get that

$$
2 R_{k-1}(\lambda)^{2}=R_{k}(\lambda)^{2},
$$

by using the eigenvalue equation and the recursive definition (3.3). 
Lemma 3.2. For all $k \geq 3$ we have

$$
\begin{aligned}
\xi\left(a_{1}\right)-\xi\left(a_{1}\right) \xi\left(a_{2}\right)-\xi\left(c_{1}\right) \xi\left(a_{2}\right) & =\xi\left(a_{k-1}\right) \xi\left(a_{k}\right), \\
\xi\left(c_{1}\right)-2 \xi\left(a_{1}\right) \xi\left(c_{2}\right) & =\xi\left(c_{k-2}\right) \xi\left(c_{k-1}\right) .
\end{aligned}
$$

Moreover for fixed $k \geq 3$ we have

$$
\xi\left(a_{i}\right) \xi\left(a_{i+1}\right)-\xi\left(a_{i+2}\right) \xi\left(a_{i-1}\right)=\xi\left(a_{k-1}\right) \xi\left(a_{k}\right)
$$

for $1 \leq i \leq k-2$.

Proof. Let $k \geq 3$ be fixed. By using (3.4) and (3.5), we get that (3.13) holds iff

$$
R_{k}(\lambda) R_{k-1}(\lambda)^{2}-R_{k-1}(\lambda)^{2} R_{k-2}(\lambda)-R_{k}(\lambda) R_{k-2}(\lambda)^{2}-\lambda R_{k-1}(\lambda)=0 .
$$

By using (3.12) we compute that this equals

$$
\lambda R_{k-1}(\lambda)\left(\frac{1}{2} R_{k}(\lambda)^{2}-R_{k}(\lambda) R_{k-2}(\lambda)-1\right) .
$$

Thus the above equation holds iff $R_{k-1}(\lambda)^{2}-R_{k}(\lambda) R_{k-2}(\lambda)-1=0$. We claim that this is in fact true for arbitrary $\lambda$, i.e. we claim that $R_{n-1}(x)^{2}-R_{n}(x) R_{n-2}(x)-1=$ $0, \forall n \geq 2$. This is shown by induction. The rest of the identities are proved along the same lines.

This concludes the proof that absolute values for a symmetric connection can be found in our examples, independent of $k$. In the case $k=4$ the absolute values can be seen in Table 4. Note that due to the equations we just solved, most of the above entries can be expressed in different ways.

3.1.4. Complex phases. Recall that we denote the complex phase of the element $z_{i j}$ by $\stackrel{\circ}{z}_{i j}$. Also recall that we had a diagonal symmetry of the form $\left|z_{i j}\right|=\left|\widetilde{z}_{j i}\right|$ for the absolute values, due to the structure of the $2 \times 2$ blocks of $U$ and of $V$. We will again take advantage of this symmetry by letting $\stackrel{\circ}{z}_{i j}=\stackrel{\circ}{z}_{j i}$ for the entries labeled by $z$. Furthermore we let $\stackrel{\circ}{\sigma}_{i j}=\stackrel{\circ}{\tau}_{j i}, \stackrel{\circ}{f}_{13}=\stackrel{\circ}{f}_{31}$, and $\stackrel{\circ}{g}_{13}=\stackrel{\circ}{g}_{31}$. This will simplify the situation by making pairs of blocks the same, hence as in the case of absolute values, this allows us to restrict our attention to the blocks of $U$. In particular we only have to deal with two $3 \times 3$ blocks.

Let us first consider the $3 \times 3$ block $A$ from $U$, which we have already determined as

$$
A=\left(\begin{array}{ccc}
\alpha f_{11} & \beta g_{11}^{\circ} & d \sigma_{11}^{\circ} \\
x g_{12} & x f_{12} & b \sigma_{12}^{\circ} \\
\circ & \circ & \circ \\
y f_{31} & a g_{31} & c \sigma_{31}
\end{array}\right),
$$

with the obvious definitions of $\alpha, \beta, d, x, b, a, y, c$. By the gauge freedom (see [4]) we can assume that $A$ has the following special form with complex phases 1 in the second row and the second column

$$
A=\left(\begin{array}{ccc}
\alpha h_{1} & \beta & d h_{2} \\
x & x & b \\
y h_{3} & a & c h_{4}
\end{array}\right),
$$

where $h_{i} \in\{z \in \mathbb{C}|| z \mid=1\}, 1 \leq i \leq 4$, that is, $h_{i}$ denotes the complex phase. We will find the complex phases for $A$, and then use these and the gauge freedom to find the complex phases for the other $3 \times 3$ block $B$, and then work through 


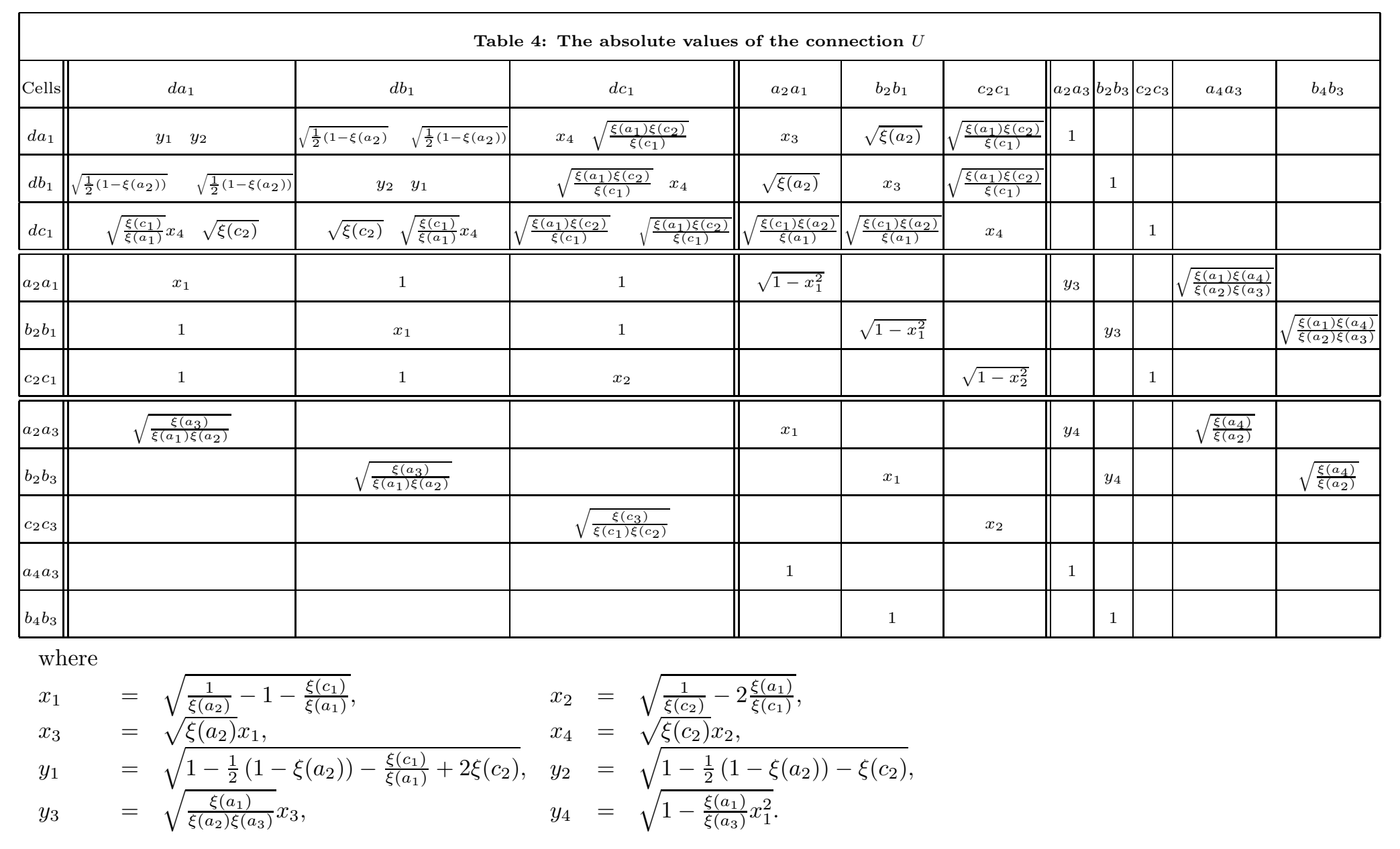


the $2 \times 2$ blocks. Set $\gamma=\frac{\frac{(b d)^{2}}{x^{2}}-\beta^{2}-\alpha^{2}}{2 \alpha \beta}, h_{1}=\gamma+i \sqrt{1-\gamma^{2}}, h_{2}=\left(-\beta x-\alpha x h_{1}\right) \frac{1}{b d}$, $h_{3}=\left(-x^{2}-\alpha \beta h_{1}\right) \frac{1}{a y}$, and $h_{4}=\left(-x a-x y h_{3}\right) \frac{1}{b c}$. Then $\left|h_{1}\right|=\left|h_{2}\right|=\left|h_{3}\right|=\left|h_{4}\right|=1$, and with these complex phases $A$ is unitary. Using the gauge choice, we know that $A$ of the form

$$
A=\left(\begin{array}{ccc}
\gamma_{1} \delta_{1} h_{1} \alpha & \gamma_{1} \delta_{2} \beta & \gamma_{1} \delta_{3} h_{2} d \\
\gamma_{2} \delta_{1} x & \gamma_{2} \delta_{2} x & \gamma_{2} \delta_{3} b \\
\gamma_{3} \delta_{1} h_{3} y & \gamma_{3} \delta_{2} a & \gamma_{3} \delta_{3} h_{4} c
\end{array}\right)
$$

is unitary, where $\gamma_{1}, \gamma_{2}, \gamma_{3}, \delta_{1}, \delta_{2}, \delta_{3} \in\{z \in \mathbb{C}|| z \mid=1\}$ are arbitrary.

Note that we had a choice above whether to use $h_{1}=\gamma+i \sqrt{1-\gamma^{2}}$ or $h_{1}=$ $\gamma-i \sqrt{1-\gamma^{2}}$, i.e. of whether to use $h_{1}$ or $\overline{h_{1}}$. If we use $\overline{h_{1}}$, then we also get the complex conjugates of the other entries of the matrix $A$. This choice does not correspond to the gauge freedom.

Consider the $3 \times 3$ block $B$, which we have previously determined as

$$
B=\left(\begin{array}{ccc}
\sqrt{1-2 \frac{\xi\left(a_{1}\right) \xi\left(c_{2}\right)}{\xi\left(c_{1}\right)}} f_{13}^{\circ} & \sqrt{\frac{\xi\left(a_{1}\right) \xi\left(c_{2}\right)}{\xi\left(c_{1}\right)}} g_{13}^{\circ} & \sqrt{\frac{\xi\left(a_{1}\right) \xi\left(c_{2}\right)}{\xi\left(c_{1}\right)}} \sigma_{13}^{\circ} \\
\sqrt{\frac{\xi\left(a_{1}\right) \xi\left(c_{2}\right)}{\xi\left(c_{1}\right)}} g_{13} & \sqrt{1-2 \frac{\xi\left(a_{1}\right) \xi\left(c_{2}\right)}{\xi\left(c_{1}\right)}} f_{13} & \sqrt{\frac{\xi\left(a_{1}\right) \xi\left(c_{2}\right)}{\xi\left(c_{1}\right)}} \sigma_{13}^{\circ} \\
\sqrt{\frac{\xi\left(a_{1}\right) \xi\left(c_{2}\right)}{\xi\left(c_{1}\right)}} f_{33} & \sqrt{\frac{\xi\left(a_{1}\right) \xi\left(c_{2}\right)}{\xi\left(c_{1}\right)}} f_{33} & \sqrt{1-2 \frac{\xi\left(a_{1}\right) \xi\left(c_{2}\right)}{\xi\left(c_{1}\right)}} \sigma_{33}^{\circ}
\end{array}\right) .
$$

Using the complex phases from the matrix $A$, and $\stackrel{\circ}{f}_{13}=\stackrel{\circ}{f}_{31}, \stackrel{\circ}{g}_{13}=\stackrel{\circ}{g}_{31}$, we see that $B$ has the special form

$$
B=\left(\begin{array}{ccc}
\theta \gamma_{3} \delta_{1} h_{3} & w \gamma_{3} \delta_{2} & w \sigma_{13}^{\circ} \\
w \gamma_{3} \delta_{2} & \theta \gamma_{3} \delta_{1} h_{3} & w \sigma_{13}^{\circ} \\
\circ & \circ \\
w f_{33} & w f_{33} & \theta \sigma_{33}^{\circ}
\end{array}\right)
$$

where $\omega$ and $\theta$ are the absolute values. We can transform this by using the gauge freedom to

$$
\left(\begin{array}{cccc}
\theta \gamma_{3} \delta_{1} h_{3} & w \gamma_{3} \delta_{2} & w \\
w \gamma_{3} \delta_{2} & \theta \gamma_{3} \delta_{1} h_{3} & w & \\
w & w & \theta \sigma_{33}^{\circ} f_{33} & \sigma_{13}^{-1}
\end{array}\right)=\left(\begin{array}{ccc}
\theta k_{1} & w k_{2} & w \\
w k_{2} & \theta k_{1} & w \\
w & w & \theta k_{3}
\end{array}\right) .
$$

Set $h_{5}=\delta_{1} \bar{\delta}_{2} h_{3}=-\frac{w}{2 \theta}+i \sqrt{1-\frac{w^{2}}{4 \theta^{2}}}, k_{1}=h_{5}^{2}, k_{2}=h_{5}$, and $k_{3}=1$ (this choice comes from the gauge freedom). Then $\left|k_{1}\right|=\left|k_{2}\right|=\left|k_{3}\right|=1$ and the matrix $B$ is unitary. Set $\sigma_{13}^{\circ}=\sigma_{33}^{\circ}=f_{33}^{\circ}=1, \gamma_{3}=1, \delta_{2}=h_{5}, \delta_{1}=h_{5}^{2} \overline{h_{3}}$. Then we have determined the elements $\gamma_{3}, \delta_{1}$, and $\delta_{2}$ occurring in $A$. Note that only $\gamma_{1}, \gamma_{2}$ and $\delta_{3}$ are now free variables of absolute value one. This means that we have determined complex phases such that all our $3 \times 3$ blocks are unitary.

Now let us work with the $2 \times 2$ blocks. Recall that by symmetry we only need to work with the $2 \times 2$ blocks from either $U$ or $V$. Thus let us consider

$$
A_{1}=\left(\begin{array}{cc}
\sqrt{\frac{1}{\xi\left(a_{2}\right)}-1-\frac{\xi\left(c_{1}\right)}{\xi\left(a_{1}\right)}} \gamma_{1} \delta_{3} h_{2} & \sqrt{\frac{\xi\left(a_{3}\right)}{\xi\left(a_{1}\right) \xi\left(a_{2}\right)}} z_{44}^{\circ} \\
\sqrt{\frac{1}{\xi\left(a_{2}\right)}-1-\frac{\xi\left(c_{1}\right)}{\xi\left(a_{1}\right)}} z_{71} & \sqrt{\frac{\xi\left(a_{3}\right)}{\xi\left(a_{1}\right) \xi\left(a_{2}\right)}} z_{74}^{\circ}
\end{array}\right)
$$


by the previous computations. Set $\delta_{3}=1, \gamma_{1}=\bar{e}_{2}, \stackrel{\circ}{z_{44}}=z_{17}^{\circ}=1, \stackrel{\circ}{z_{47}}=-1$; then $A_{1}$ is unitary. Now

$$
A_{2}=\left(\begin{array}{cc}
\sqrt{\frac{1}{\xi\left(c_{2}\right)}-2 \frac{\xi\left(a_{1}\right)}{\xi\left(c_{1}\right)}} & \sqrt{\frac{\xi\left(c_{3}\right)}{\xi\left(c_{1}\right) \xi\left(c_{2}\right)}} z_{66}^{\circ} \\
\sqrt{\frac{\xi\left(c_{3}\right)}{\xi\left(c_{1}\right) \xi\left(c_{2}\right)}} z_{93}^{\circ} & \sqrt{\frac{1}{\xi\left(c_{2}\right)}-2 \frac{\xi\left(a_{1}\right)}{\xi\left(c_{1}\right)}} z_{96}^{\circ}
\end{array}\right) .
$$

Thus set $z_{66}^{\circ}=z_{93}^{\circ}=1, z_{96}^{\circ}=-1$; then $A_{2}$ is unitary. This way we can work our way down towards the lower right corner of Table 1 by letting each $2 \times 2$ block have three elements with complex phase 1 and one element with complex phase -1 . This concludes the construction of the symmetric connection. In the case of $k=4$, the complex phases are given in Table 5, where $\theta_{1}=h_{1} \overline{h_{2} h_{3}} h_{5}^{2}, \theta_{2}=\overline{h_{2}} h_{5}, \theta_{3}=\overline{h_{3}} h_{5}^{2}$ and $h_{1}, h_{2}, h_{3}, h_{4}$ and $h_{5}$ are as above.

\begin{tabular}{|c|c|c|c|c|c|c|c|c|c|c|c|}
\hline \multicolumn{12}{|c|}{ Table 5: Complex phases of the connection $U$} \\
\hline Cells & $d a_{1}$ & $d b_{1}$ & $d c_{1}$ & $a_{2} a_{1}$ & $b_{2} b_{1}$ & $c_{2} c_{1}$ & $a_{2} a_{3}$ & $b_{2} b_{3}$ & $c_{2} c_{3}$ & $a_{4} a_{3}$ & $b_{4} b_{3}$ \\
\hline$d a_{1}$ & $\theta_{1} \theta_{2}$ & $h_{5} \theta_{3}$ & $h_{5}^{2} h_{5}$ & 1 & 1 & 1 & 1 & & & & \\
\hline$d b_{1}$ & $\theta_{3} h_{5}$ & $\theta_{2} \theta_{1}$ & $h_{5} h_{5}^{2}$ & 1 & 1 & 1 & & 1 & & & \\
\hline$d c_{1}$ & $h_{5}^{2} h_{5}$ & $h_{5} h_{5}^{2}$ & 11 & $h_{4}$ & $h_{4}$ & 1 & & & 1 & & \\
\hline$\overline{\overline{a_{2} a_{1}}}$ & $\begin{array}{ll}1 \\
\end{array}$ & $\begin{array}{ll}1 \\
\end{array}$ & 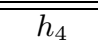 & 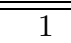 & & & -1 & & & 1 & \\
\hline$b_{2} b_{1}$ & 1 & 1 & $h_{4}$ & & 1 & & & -1 & & & 1 \\
\hline$c_{2} c_{1}$ & 1 & 1 & 1 & & & 1 & & & -1 & & \\
\hline$a_{2} a_{3}$ & 1 & & & -1 & & & 1 & & & 1 & \\
\hline$b_{2} b_{3}$ & & 1 & & & -1 & & & 1 & & & 1 \\
\hline$c_{2} c_{3}$ & & & 1 & & & -1 & & & & & \\
\hline$a_{4} a_{3}$ & & & & 1 & & & 1 & & & & \\
\hline$b_{4} b_{3}$ & & & & & 1 & & & 1 & & & \\
\hline
\end{tabular}

3.2. The result. From the commuting square (B.1) we construct both the horizontal and the vertical subfactors as explained in section 2. They are irreducible due to Wenzl's dimension estimate [20, since the graph $\Gamma_{k}$ in Figure 2 has a vertex, which is only connected to one other vertex by a single edge. Moreover the vertical one, the Haagerup-Schou subfactor, has index $\left\|G_{k}\right\|^{2}$. These index values are between 4 and 4.5 [18. We conclude from [7], 1], and [2] that the vertical subfactors constructed from the initial commuting square (3.1) based on the graphs $\Gamma_{k}$ for $k \geq 3$ have principal graphs $A_{\infty}$. Thus in particular the Haagerup-Schou subfactors have infinite depth. This implies that the horizontal subfactor has infinite principal graph, too [17. Moreover by a result of Popa these subfactors are non-amenable, since their index is larger than the norm of the principal graph [16]. Using the notation of section 2 what we have achieved is summarized in the following.

Theorem 3.3. Let $\Gamma_{k}$ denote the graph in Figure 2 and consider the non-degenerate commuting square

$$
\begin{array}{ccccc} 
& & G_{k} & & \\
G_{k} G_{k}^{t}-I & A_{10} & \subset & A_{11}, \tau & \\
& \cup & & \cup & G_{k}^{t} G_{k}-I \\
& A_{00} & \subset & A_{01} & \\
& & G_{k} & &
\end{array}
$$

where the trace $\tau$ on $A_{11}$ is the normalized Markov trace and $A_{00}$ is abelian. Let $\phi$ be the graph automorphism of $\Gamma_{k}$, which switches the a-ray and the b-ray and the 
double d-d edge in $G_{k} G_{k}^{t}-I$ as described above. In particular $\phi$ is non-trivial on the set of vertices. Let $k \geq 3$. Then the commuting square (3.1) has a symmetric connection with respect to $\phi$. Let $N \subset M$ denote either the vertical or the horizontal subfactor constructed from (3.1) and define $\varphi_{11}$ as in Definition 2.8. Then $\varphi_{11}$ is a commuting square automorphism, hence extends to an automorphism $\varphi$ of both the horizontal and the vertical subfactors. Moreover $\varphi$ is not inner, i.e. $\varphi$ is not of the form $\operatorname{Ad}(u)$ for $u \in \mathcal{U}(N)$.

Proof. Let $k \geq 3$. For this series of examples we have explicitly constructed a symmetric connection, hence the result follows from Theorem 2.17 .

\section{ACKNOWLEDGMENTS}

This paper is based on the author's Ph.D. thesis at University of California, Santa Barbara, under the supervision of Professor Dietmar Bisch, whom we thank for guidance and advice. The work was supported by a fellowship from The Danish Natural Science Research Council, by NSF grant DMS-9877067, and by a fellowship from the UCSB Graduate Division.

\section{REFERENCES}

[1] Asaeda, M. and Haagerup, U. (1999). Exotic subfactors of finite depth with Jones indices $(5+\sqrt{13}) / 2$ and $(5+\sqrt{17}) / 2$. Communications in Mathematical Physics, 202, 1-63. MR 2000c: 46120

[2] Bisch, D. (1998). Principal graphs of subfactors with small Jones index. Mathematische Annalen, 311, 223-231. MR 2000k:46087

[3] Bratteli, O. (1972). Inductive limits of finite dimensional $C^{*}$-algebras. Transactions of the American Mathematical Society, 171, 195-234. MR 47:844

[4] Evans, D. E. and Kawahigashi, Y. (1998). Quantum symmetries on operator algebras. Oxford University Press. MR 99m:46148

[5] Goodman, F., de la Harpe, P. and Jones, V. F. R. (1989). Coxeter graphs and towers of algebras. MSRI Publications (Springer), 14. MR 91c:46082

[6] Goto, S. (1995). Symmetric flat connections, triviality of Loi's invariant, and orbifold subfactors. Publications of the RIMS, Kyoto University, 31, 609-624. MR 97e:46081

[7] Haagerup, U. (1994). Principal graphs of subfactors in the index range $4<3+\sqrt{2}$. in Subfactors - Proceedings of the Taniguchi Symposium, Katata -, (ed. H. Araki, et al.), World Scientific, 1-38. MR 96d:46081

[8] Jones, V. F. R. (1983). Index for subfactors. Inventiones Mathematicae, 72, 1-25. MR 84d:46097

[9] Jones, V. F. R. and Sunder, V. S. (1997). Introduction to subfactors. London Math. Soc. Lecture Notes Series 234, Cambridge University Press. MR 98h:46067

[10] Kawahigashi, Y. (1995). On flatness of Ocneanu's connections on the Dynkin diagrams and classification of subfactors. Journal of Functional Analysis, 127, 63-107. MR 95j:46075

[11] Kawahigashi, Y. (1993). Centrally trivial automorphisms and an analogue of Connes's $\chi(M)$ for subfactors. Duke Mathematical Journal, 71, 93-118. MR 94k:46131

[12] Loi, P. H. (1996). On automorphisms of subfactors. Journal of Functional Analysis, 141, 275-293. MR 98b:46082

[13] Ocneanu, A. (1988). Quantized group, string algebras and Galois theory for algebras. Operator algebras and applications, Vol. 2 (Warwick, 1987), (ed. D. E. Evans and M. Takesaki), London Mathematical Society Lecture Note Series Vol. 136, Cambridge University Press, 119-172. MR 91k:46068

[14] Ocneanu, A. (1991). Quantum symmetry, differential geometry of finite graphs and classification of subfactors, University of Tokyo Seminary Notes 45 (Notes recorded by Kawahigashi, Y.).

[15] Popa, S. (1983). Orthogonal pairs of $*$-subalgebras in finite von Neumann algebras. Journal of Operator Theory, 9, 253-268. MR 84h:46077 
[16] Popa, S. (1994). Classification of amenable subfactors of type II. Acta Mathematica, 172, 163-255. MR 95f:46105

[17] Sato, N. (1997). Two subfactors arising from a non-degenerate commuting square - An answer to a question raised by V. F. R. Jones-. Pacific Journal of Mathematics, 180, 369-376. MR 99c: 46073

[18] Schou, J. (1990). Commuting squares and index for subfactors. Ph.D. thesis at Odense University.

[19] Svendsen, A.L. (2002). Commuting squares and automorphisms of subfactors. Ph.D. thesis at University of California at Santa Barbara.

[20] Wenzl, H. (1988). Hecke algebras of type $A_{n}$ and subfactors. Inventiones Mathematicae, 92, 345-383. MR 90b:46118

Department of Mathematics, University of Oslo, P.O. Box 1053, Blindern, N - 0316 OSLO, NORWAY

E-mail address: annelsv@math.uio.no

Current address: Department of Mathematics, University of Copenhagen, Universitetsparken 5, DK-2100 Copenhagen 0, Denmark

E-mail address: svendsen@math.ku.dk 